TRANSACTIONS OF THE

AMERICAN MATHEMATICAL SOCIETY

Volume 361, Number 5, May 2009, Pages 2755-2788

S 0002-9947(08)04823-X

Article electronically published on December 17, 2008

\title{
SCALAR PARABOLIC PDES AND BRAIDS
}

\author{
R. W. GHRIST AND R. C. VANDERVORST
}

\begin{abstract}
The comparison principle for scalar second order parabolic PDEs on functions $u(t, x)$ admits a topological interpretation: pairs of solutions, $u^{1}(t, \cdot)$ and $u^{2}(t, \cdot)$, evolve so as to not increase the intersection number of their graphs. We generalize to the case of multiple solutions $\left\{u^{\alpha}(t, \cdot)\right\}_{\alpha=1}^{n}$. By lifting the graphs to Legendrian braids, we give a global version of the comparison principle: the curves $u^{\alpha}(t, \cdot)$ evolve so as to (weakly) decrease the algebraic length of the braid.

We define a Morse-type theory on Legendrian braids which we demonstrate is useful for detecting stationary and periodic solutions to scalar parabolic PDEs. This is done via discretization to a finite-dimensional system and a suitable Conley index for discrete braids.

The result is a toolbox of purely topological methods for finding invariant sets of scalar parabolic PDEs. We give several examples of spatially inhomogeneous systems possessing infinite collections of intricate stationary and time-periodic solutions.
\end{abstract}

\section{INTRODUCTION}

We consider the invariant dynamics of one-dimensional second order parabolic equations of the type

$$
u_{t}=u_{x x}+g\left(x, u, u_{x}\right)
$$

where $u$ is a scalar function of the variables $t \in \mathbb{R}$ (time) and $x \in S^{1}=\mathbb{R} / \ell \mathbb{Z}$ (periodic boundary conditions in space), and $g$ is a $C^{1}$-function of its arguments.

1.1. Assumptions. The case of periodic boundary conditions in $x$ provides richer dynamics in general than Neumann or Dirichlet boundary conditions; however, the techniques we introduce are applicable to a surprisingly large variety of nonlinear boundary conditions. Our techniques come from the finite-dimensional theory of parabolic systems 22] where topological invariants are introduced to find various types of solutions. Parabolic equations such as Eqn. (1.1) allow the same invariants as in the finite-dimensional theory via standard discretization schemes.

This paper does not deal with the initial value problem, but rather with the bounded invariant dynamics: bounded solutions of Eqn. (1.1) that exist for all time $t$. One distinguishes three types of behaviors which are the building blocks of all

Received by the editors November 11, 2004 and, in revised form, December 14, 2007.

2000 Mathematics Subject Classification. Primary 35K90, 37B30.

The first author was supported in part by NSF PECASE grant DMS-0337713.

The second author was supported by NWO VIDI grant 639.032.202.

These results were announced in 21 . 
bounded invariant solutions to Eqn. (1.1) [5, 15, 24, 35]:

(i) stationary patterns: $u(t, x)=u(x), \forall t \in \mathbb{R}$,

(ii) periodic motions: $u(t+T, x)=u(t, x)$, for some period $T>0$,

(iii) homoclinic/heteroclinic connections: $\lim _{t \rightarrow \pm \infty} u(t, x)=u_{ \pm}(x)$, where $u_{ \pm}$ are stationary or periodic solutions of Eqn. (1.1).

For the remainder of this paper we impose one or two natural assumptions on Eqn. (1.1). The first hypothesis is a subquadratic growth condition on the $u_{x}$ term of $g$ :

(g1) There exist constants $C>0$ and $0<\gamma<2$, such that $|g(x, u, v)| \leq$ $C\left(1+|v|^{\gamma}\right)$, uniformly in both $x \in S^{1}$ and on compact intervals in $u$.

This will be necessary for regularity and control of derivatives of solution curves; cf. [5. This condition is sharp: one can find examples of $g$ with quadratic growth in $u_{x}$ for which solutions have singularities in $u_{x}$. Since our topological data are drawn from graphs of $u$, the bounds on $u$ need to imply bounds on $u_{x}$ and $u_{x x}$ : (g1) does just that.

A second gradient hypothesis will sometimes be assumed:

$$
g \text { is exact, i.e., }
$$

$$
u_{x x}+g\left(x, u, u_{x}\right)=a\left(x, u, u_{x}\right)\left[\frac{d}{d x} \partial_{u_{x}} L-\partial_{u} L\right]
$$

for a strictly positive and bounded function $a=a\left(x, u, u_{x}\right)$ and some Lagrangian $L$ satisfying $a\left(x, u, u_{x}\right) \cdot \partial_{u_{x}}^{2} L\left(x, u, u_{x}\right)=1$.

In this case, we have a gradient system whose stationary solutions are critical points of the action $\int L\left(x, u, u_{x}\right) d x$ over loops of integer period in $x$. This condition holds for a wide variety of systems. For the invariant dynamics possibility, (ii) is then excluded as well as the existence of homoclinic connections. In general, systems with Neumann or Dirichlet boundary conditions admit a gradient-like structure: there exists a Lyapunov function which decreases strictly in $t$ along nonstationary orbits, which also precludes the existence of nonstationary time-periodic solutions. It was shown by Zelenyak [35] that this gradient-like condition holds for many nonlinear boundary conditions which are a mixture of Dirichlet and Neumann.

1.2. Lifting the comparison principle. An important property of one-dimensional parabolic dynamics is the lap-number principle of Sturm, Matano, and Angenent [2, 27, 32] which, roughly, states that the number of nodal regions in $x$ of $u(t, x)$ is a weak Lyapunov function for Eqn. (1.1). There exists a huge literature on applications of the lap-number principle to the dynamics of Eqn. (1.1). For instance, in the work of Fiedler and Rocha [17, 18, 19] the lap-number principle in used in a novel manner to completely describe the dynamics of the attractors for many parabolic equations such as Eqn. (1.1). In this context we also mention the work of Fiedler and collaborators on for example connecting orbits for parabolic dynamics [9, 10, 11]. See also the related works [1, 6, 25] for a sampling of lapnumber methods. We will use the lap-number principle on a more topological level, i.e. forcing of solutions via topological invariants. This will lead to a very general and robust "forcing theory" for invariant sets.

The lifting of this principle to the simultaneous evolution of pairs of solutions is extremely fruitful. Consider two solutions $u^{1}(t, x)$ and $u^{2}(t, x)$. Any tangency between the graphs $u^{1}(t, \cdot)$ and $u^{2}(t, \cdot)$ at time $t=t_{*}$ is removed for $t=t_{*}+\epsilon$ (for 
all small $\epsilon>0$ ) so as to strictly decrease the number of intersections of the graphs. This holds even for highly degenerate tangencies of curves [2]. As shown in the work of Fiedler and Mallet-Paret [15], this comparison principle implies that the dynamics of Eqn. (1.1) is weakly Morse-Smale (all bounded orbits are either fixed points, periodic orbits, or connecting orbits between these); see [24, 35].

The idea behind this paper, following the discrete version of this phenomenon in 22 , is to "lift" the comparison principle from pairs of solutions to larger ensembles of solution curves. The local data attached to pairs of curves - intersection number - can be lifted to more global data about patterns of intersections via the language of topological braid theory. A similar theory for geodesics on two-dimensional surfaces has been developed in [3], and has served as an inspiration for some of the ideas used here.

Consider a collection $\mathbf{u}=\left\{u^{\alpha}(t, \cdot)\right\}_{\alpha=1}^{n}$ of $n>1$ solutions to Eqn. (1.1), where, to obey the periodic boundary conditions in $x,\left\{u^{\alpha}(t, 0)\right\}_{\alpha=1}^{n}=\left\{u^{\alpha}(t, 1)\right\}_{\alpha=1}^{n}$ as sets of points 1 Instead of thinking of the graphs of $u^{\alpha}(t, \cdot)$ as being evolving curves in the $(x, u)$-plane, we take the 1-jet extension of each curve and think of it as an evolving curve in $\left(x, u, u_{x}\right)$-space. Specifically, for each $t, u^{\alpha}(t, \cdot):[0,1] \rightarrow[0,1] \times \mathbb{R}^{2}$ is given by $x \mapsto\left(x, u^{\alpha}(x, t), u_{x}^{\alpha}(x, t)\right)$. As long as these curves do not intersect in their $3-\mathrm{d}$ representations, we have what is commonly referred to as a braid. In particular, such a braid is said to be closed (the ends $x=0$ and $x=1$ are identified) and Legendrian (the curves are all tangent to the standard contact structure $d x_{2}-x_{3} d x_{1}=0$ ).

As these curves evolve under the PDE, the topological type of the braid can change. The topological equivalence class of a closed Legendrian braid is the appropriate analogue of the intersection data for pairs of curves. Indeed, there is a natural group structure on braids with $n$ strands. We argue in a "braid-theoretic" version of the comparison principle that the algebraic length of a braid given by solutions $\left\{u^{\alpha}(t)\right\}_{\alpha=1}^{n}$ is a weak Lyapunov function for the dynamics of Eqn. (1.1).

1.3. Main results. The goal of this paper, following earlier work in [22] on a discrete version of this problem, is to define an index for closed Legendrian braids and to use this as the basis for detecting invariant dynamics of Eqn. (1.1). See $₫ 2$ for definitions and background on the discrete version.

For purposes of detecting invariant dynamics of Eqn. (1.1), we work with braids $\mathbf{u}$ relative to some fixed braid $\mathbf{v}$. One thinks of $\mathbf{v}$ as a braid for which dynamical information is known, namely, that its strands are $t$-invariant solutions to Eqn. (1.1), the entire set of which respects the periodic boundary conditions (individual strands might not; see Figure 1). One thinks of $\mathbf{u}$ as consisting of "free" strands about which nothing is known with regards to dynamical behavior.

We show that there exists a well-defined homotopy index that maps a (closed, Legendrian, relative) braid class represented by $\{\mathbf{u}$ REL $\mathbf{v}\}$ to a pointed homotopy class of spaces, $\mathbf{H}(\mathbf{u}$ REL $\mathbf{v})$. This index is at heart a Conley index for a suitable configuration space which is isolated thanks to the braid-theoretic comparison principle. A coarser homology index sends such braids to a polynomial $P_{\tau}(\mathbf{H})$ in one variable, $\tau$.

The main results of this paper are forcing theorems for stationary and periodic solutions.

\footnotetext{
${ }^{1}$ This condition permits solutions with integral period which "wrap" around the circle.
} 
1.3.1. Stationary solutions. For our main results we restrict to braid classes which have two compactness properties: proper and bounded. Roughly speaking, a relative braid class $\{\mathbf{u}$ REL $\mathbf{v}\}$ is proper if none of the components of $\mathbf{u}$ can be collapsed onto $\mathbf{u}$ or $\mathbf{v}$. A relative braid class $\{\mathbf{u}$ REL $\mathbf{v}\}$ is bounded if all strands of $\mathbf{u}$ are uniformly bounded with respect to all representatives $\mathbf{u}$ REL $\mathbf{v}$ of the braid class; see $₫ 2$

Theorem 1.1. Let Eqn. (1.1) satisfy (g1) with $\mathbf{v}$ a stationary braid. If $\{\mathbf{u}$ REL $\mathbf{v}\}$ is a bounded proper braid class, then there exists a stationary solution of this braid class if the Euler characteristic of $\mathbf{H}, \chi(\mathbf{H}):=P_{-1}(\mathbf{H})$, is nonvanishing.

In the case of an exact system the Poincaré polynomial of $\mathbf{H}$ provides a stronger result.

Theorem 1.2. If $g$ satisfies both $(\mathbf{g} 1)$ and $(\mathbf{g} 2)$, then $P_{\tau}(\mathbf{H}) \neq 0$ implies that there exists a stationary solution in the desired braid class.

The above theorem is formulated for periodic boundary conditions. In the case of most other nonperiodic boundary conditions the result by Zelenyak 35] implies that Eqn. (1.1) is automatically gradient-like so that Theorem 1.2 automatically holds in that case (without assuming (g2)).

Remark 1.3. The result for obtaining stationary solutions to Eqn. (1.1), i.e. solutions of $u_{x x}+g\left(x, u, u_{x}\right)=0$, immediately implies the same result for fully nonlinear equations of the form

$$
f\left(x, u, u_{x}, u_{x x}\right)=0,
$$

where $f$ satisfies the following hypotheses: (i) $0<\lambda \leq \partial_{w} f(x, u, v, w) \leq \lambda^{-1}$, uniformly for all $(x, u, v, w) \in S^{1} \times \mathbb{R}^{3}$, and (ii) $|f(x, u, v, w)| \leq C\left(1+|v|^{\gamma}\right)$, uniformly in both $x \in S^{1}$ and on compact intervals in $u$ and $w$, for some $0<\gamma<2$. This can be achieved by solving the equation $f(x, u, v, w)=0$ with respect to $w$, which yields a unique solution $w=-g\left(x, u, u_{x}\right)$. Using the Implicit Function Theorem one easily deduces that $g$ satisfies Hypothesis (g1), and thus $f\left(x, u, u_{x}, u_{x x}\right)=0$ is equivalent to $u_{x x}+g\left(x, u, u_{x}\right)=0$.

Remark 1.4. Obtaining multiplicity results in the exact case requires a more careful analysis of the limiting procedure. However, by using a different method of discretization we can easily obtain multiplicity results in various simplified cases. For example if we consider equations of the form

$$
u_{x x}+g(x, u)=0,
$$

where $g$ is a $C^{1}$-function of $u$, we obtain the following result.

Theorem 1.5. Let $\mathbf{v}$ be a stationary skeleton for Eqn. (1.3), and let $\{\mathbf{u}$ REL $\mathbf{v}\}$ be a proper and bounded braid class. Then Eqn. (1.3) has at least $\left|P_{\tau}(\mathbf{H})\right|$ stationary solutions in $\{\mathbf{u}$ REL $\mathbf{v}\}$, where $|\cdot|$ denotes the number of nonzero monomials.

In Appendix D we will give a proof of this result using the method of broken geodesics. It remains an interesting question to see if the analogue of this theorem can be proved for Eqn. (1.1) using the discretization approach employed in this paper.

Remark 1.6. In the exact case above the lower bound on the number of critical points can refined even further. For parabolic recurrence relations the spectrum 
of a critical point satisfies $\lambda_{0}<\lambda_{1} \leq \lambda_{2}<\lambda_{3} \leq \lambda_{4}<\lambda_{5} \ldots$. This ordering has a special bearing on nondegenerate critical points with odd index. To be more precise, for a 'topological' non-degenerate critical point $\mathbf{u}$ with $P_{\tau}(\mathbf{u})=A \tau^{2 k+1}$ $(A>0)$ it holds that $A=1$. More details of this can be found in the work of Dancer 14. As a direct consequence there are at least as many critical points as the sum of the odd Betti numbers of $\mathbf{H}$. If we write $P_{\tau}(\mathbf{H})=P_{\tau}^{\text {odd }}(\mathbf{H})+P_{\tau}^{\text {even }}(\mathbf{H})$, then our lower bound on the number of critical points becomes

$$
\left\|P_{\tau}(\mathbf{H})\right\|:=P_{1}^{\text {odd }}(\mathbf{H})+\left|P_{\tau}^{\text {even }}(\mathbf{H})\right|,
$$

which lies in between $\left|P_{\tau}(\mathbf{H})\right|$ and $P_{1}(\mathbf{H})$. In some cases these bounds can be strengthened even more. For instance if we consider fixed (linear) boundary conditions, then $\lambda_{0}<\lambda_{1}<\lambda_{2}<\lambda_{3}<\lambda_{4}<\lambda_{5} \ldots$. Consequently, for any topologically nondegenerate critical point with $P_{\tau}(\mathbf{u})=A \tau^{k}(A>0)$ it then holds that $A=1$, which implies that $P_{1}(\mathbf{H})$ is a lower bound for the number of critical points - the same as one would obtain in the nondegenerate case.

The proof of Theorem 1.1 appears in \$7. First, however, we introduce the relevant portions of braid theory (\$2), followed by a review ( $\$ \S 3$ 4 $)$ of the discrete braid index constructed in 22, and a definition of the invariant $\mathbf{H}$ for proper and bounded relative braid classes $\{\mathbf{u}$ REL $\mathbf{v}\}$.

This theory applies to a wide array of inhomogeneous equations. In $\$ 5$ we show:

Example 1.7. The equation

$$
u_{t}=u_{x x}-\frac{5}{8} \sin 2 x u_{x}+\frac{\cos x}{\cos x+\frac{3}{\sqrt{5}}} u\left(u^{2}-1\right)
$$

possesses stationary solutions in an infinite number of distinct braid classes. We also show that one can embed a Bernoulli shift into the stationary equation.

Example 1.8. For any $\epsilon \ll 1$ and any smooth nonconstant $h: S^{1} \rightarrow(0,1)$, the equation

$$
\epsilon^{2} u_{t}=\epsilon^{2} u_{x x}+h(x) u\left(1-u^{2}\right)
$$

possesses stationary solutions spanning an infinite collection of braid classes, and thus infinitely many geometrically distinct periodic solutions of integer period. This example was studied by Nakashima [28, 29].

The former example is 'artificial' and generated for ease of computing examples of the homotopy braid index. The latter example is an inhomogeneous ChafeeInfante equation of general interest. Both these examples can be generalized greatly. Theorem 5.4 gives extremely broad conditions which force an infinite collection of stationary solutions.

1.3.2. Periodic solutions. We also lay the foundation for using the braid index to find time-periodic solutions. Under Hypothesis (g1) we prove an analogue of Theorem 1.1 for time-periodic solutions of Eqn. (1.1). As we pointed out before, time-periodic solutions can exist by the grace of the boundary conditions. As the result of Zelenyak implies, in most cases a weak version of (g2) holds (gradient-like) and the only critical elements are stationary solutions.

Remark 1.9. A fundamental class of time-periodic orbits are the so-called rotating waves. For an equation which is autonomous in $x$, one makes the rotating wave 
hypothesis that $u(t, x)=U(x-c t)$, where $c$ is the unknown wave speed. Stationary solutions for the resulting equation on $U(\xi)$ yield rotating waves. Modulo the unknown wave speed - a nonlinear eigenvalue problem — Theorem 1.1now applies. In 5] it was proved that time-periodic solutions are necessarily rotating waves for an equation autonomous in $x$. However, in the nonautonomous case, there is an extraordinary richness of permissible dynamics. We introduce a very general technique for finding time-periodic solutions without the rotating wave hypothesis.

We present a very general technique for finding time-periodic solutions without the rotating wave hypothesis.

Theorem 1.10. Let Eqn. (1.1) satisfy (g1) with $\mathbf{v}$ a stationary braid. Let $\{\mathbf{u}$ REL $\mathbf{v}\}$ be a bounded proper braid class with $\mathbf{u}$ a single-component braid and $P_{\tau}(\mathbf{H}) \neq 0$. If Eqn. (1.1) does not contain stationary braids in this braid class, then there exists a time-periodic solution in this braid class.

Remark 1.11. In certain examples one can find braid classes in which a given equation cannot have stationary solutions. Since the only possible critical elements in that case are periodic orbits it follows that the Poincaré polynomial has to be of the form $P_{\tau}(\mathbf{H})=(1+\tau) p_{\tau}(\mathbf{H})$. For free strands forming multi-component braids $\mathbf{u}$, each component of $\mathbf{u}$ will be time-periodic. Their periods may not be rationally related, however, leading to a quasi-periodic solution in time in the multi-component braid class.

It was shown in [5] that a singularly perturbed van der Pol equation,

$$
u_{t}=\epsilon u_{x x}+u\left(1-\delta^{2} u^{2}\right)+u_{x} u^{2},
$$

possesses rotating waves for each fixed value of $\epsilon \ll 1$ and $0<\delta$, with that number tending to infinity as $\epsilon \rightarrow 0$. We generalize their result:

Example 1.12. Consider the equation

$$
u_{t}=u_{x x}+u b(u)+u_{x} h\left(x, u, u_{x}\right)
$$

where the nonlinearity is assumed to satisfy (g1), i.e. $h$ has sublinear growth in $u_{x}$ at infinity. Moreover, $b$ and $h$ satisfy the following hypotheses:

(b1) $b(0)>0$, and $b$ has at least one positive and one negative root;

(b2) $h(x, 0,0)=0$, and $h>0$ on $\left\{u u_{x} \neq 0\right\}$.

Then this equation possesses time-periodic solutions spanning an infinite collection of braid classes. The condition $h(x, 0,0)$ is imposed here to simplify technicalities, and the same result should hold under milder conditions.

We provide details in $\$ 6$. The set of periodic solutions implied are dynamically unstable and have unbounded spatial period. In the most general case (those systems with $x$-dependence), the periodic solutions are not rigid rotating waves.

\section{BRAIDS}

The results of this paper require very little of the extensive theory of braids developed by topologists [7. However, since the definitions motivate our constructions, we give a brief tour. 
2.1. Topological braids. A topological braid on $n$ strands is an embedding $\beta$ : $\coprod_{1}^{n}[0,1] \hookrightarrow \mathbb{R}^{3}$ of a disjoint union of $n$ copies of $[0,1]$ into $\mathbb{R}^{3}$ such that

(a) the left endpoints $\beta\left(\coprod_{1}^{n}\{0\}\right)$ are $\{(0, i, 0)\}_{i=1}^{n}$;

(b) the right endpoints $\beta\left(\coprod_{1}^{n}\{1\}\right)$ are $\{(1, i, 0)\}_{i=1}^{n}$; and

(c) $\beta$ is transverse to the planes $x_{1}=$ constant.

Two braids are said to be of the same topological braid class if they are homotopic in the space of braids: one braid deforms to the other without any intersections of the strands. A closed topological braid is obtained if one quotients out the range of the braid embeddings via the equivalence relation $\left(0, x_{2}, x_{3}\right) \sim\left(1, x_{2}, x_{3}\right)$ and alters the restrictions (a) and (b) of the position of the endpoints to be $\beta\left(\coprod_{i}^{n}\{0\}\right)=$ $\beta\left(\coprod_{1}^{n}\{1\}\right)$. Thus, a closed braid is a collection of disjoint embedded arcs in $[0,1] \times$ $\mathbb{R}^{2}$ (with periodic boundary conditions in the first variable) which are everywhere transverse to the planes $x_{1}=$ constant.

In this paper, we restrict attention to those braids whose strands are of the form $\left(x, u(x), u_{x}(x)\right)$ for $0 \leq x \leq 1$. These are sometimes called Legendrian braids as they are tangent to the canonical contact structure $d x_{2}-x_{3} d x_{1}$. No knowledge of Legendrian braid theory is assumed for the remainder of this work, but we will use the term freely to denote those braids lifted from graphs.

2.2. Braid diagrams. The specification of a topological braid class (closed or otherwise) may be accomplished unambiguously by a labeled projection to the $\left(x_{1}, x_{2}\right)$-plane: a braid diagram. Labeling is done as follows: perturb the projected curves slightly so that all strand crossings in the projection are transversal and disjoint. Then, mark each crossing via $(+)$ or $(-)$ to indicate whether the crossing is "left over right" or "right over left" respectively.

Since a Legendrian braid is of the form $\left(x, u(x), u_{x}(x)\right)$, no such marking of crossings in the $(x, u)$ projection are necessary: all crossings have positive labels. For the remainder of this paper we will consider only such positive braid diagrams. We will analyze parabolic PDEs by working on spaces of such braid diagrams. Although Legendrian braids are the right types of braids to work with as solutions to Eqn. (1.1) (cf. the smoothing of initial data for heat flow), our discretization techniques will require a more robust $C^{0}$-theory for braid diagrams. Thus, we work on spaces of braid diagrams with topologically transverse strands:

Definition 2.1. The space of closed positive braid diagrams on $n$ strands, denoted $\Omega^{n}$, is the space of all pairs $(\mathbf{u}, \tau)$ where $\tau \in S_{n}$ is a permutation on $n$ elements, and $\mathbf{u}=\left\{u^{\alpha}(x)\right\}_{\alpha=1}^{n}$ is an unordered collection of $H^{1}$-functions - strands - satisfying the following conditions:

(a) Periodicity: $u^{\alpha}(1)=u^{\tau(\alpha)}(0)$ for all $\alpha$.

(b) Transversality: for any $\alpha \neq \alpha^{\prime}$ such that $u^{\alpha}\left(x_{*}\right)=u^{\alpha^{\prime}}\left(x_{*}\right)$ for some $x_{*} \in[0,1]$, it holds that $u^{\alpha}(x)-u^{\alpha^{\prime}}(x)$ has an isolated sign change at $x=x_{*}$.

Because the strands of $\mathbf{u}$ are unordered, we naturally identify all pairs $(\mathbf{u}, \tau)$ and $(\mathbf{u}, \tilde{\tau})$ satisfying $\tilde{\tau}=\sigma \tau \sigma^{-1}$ for some permutation $\sigma \in S_{n}$. Henceforth we suppress the permutation $\tau$ from the description of a braid, it being understood implicitly.

The path components of $\Omega^{n}$ comprise the braid classes of closed positive braid diagrams. The braid class of a braid diagram $\mathbf{u}$ is denoted by $\{\mathbf{u}\}$. Any braid 
diagram $\mathbf{u}$ with $C^{1}$-strands naturally lifts to a Legendrian braid by the 1-jet extension of $u^{\alpha}$ to the curve $\left(x, u^{\alpha}(x), u_{x}^{\alpha}(x)\right)$. If we allow the strands to intersect disregarding condition (b) of Definition 2.1 — we obtain a closure of the space $\Omega^{n}$, which we denote $\overline{\Omega^{n}}$. The 'discriminant' $\Sigma^{n}:=\overline{\Omega^{n}}-\Omega^{n}$ defines the singular braid diagrams.

2.3. Discrete braid diagrams. From topological braids we have passed to braid diagrams in order to describe invariant curves for parabolic PDEs. There is one last transformation we must impose: a spatial discretization.

Definition 2.2. The space of period d discrete braid diagrams on $n$ strands, denoted $\mathcal{D}_{d}^{n}$, is the space of all pairs $(\mathbf{u}, \tau)$ where $\tau \in S_{n}$ is a permutation on $n$ elements, and $\mathbf{u}=\left\{u^{\alpha}\right\}_{\alpha=1}^{n}$ is an unordered collection of vectors $u^{\alpha}=\left(u_{i}^{\alpha}\right)_{i=0}^{d}$, i.e. strands, satisfying the following conditions:

(a) Periodicity: $u_{d}^{\alpha}=u_{0}^{\tau(\alpha)}$ for all $\alpha$.

(b) Transversality: for any $\alpha \neq \alpha^{\prime}$ such that $u_{i}^{\alpha}=u_{i}^{\alpha^{\prime}}$ for some $i$,

$$
\left(u_{i-1}^{\alpha}-u_{i-1}^{\alpha^{\prime}}\right)\left(u_{i+1}^{\alpha}-u_{i+1}^{\alpha^{\prime}}\right)<0 .
$$

As in Definition 2.1, the permutation $\tau$ is defined up to conjugacy (since the strands are unordered) and will henceforth not be explicitly written.

The path components of $\mathcal{D}_{d}^{n}$ comprise the discrete braid classes of period $d$. The discrete braid class of a discrete braid diagram $\mathbf{u}$ is denoted $[\mathbf{u}]$. If we disregard condition (b) of Definition 2.2, we obtain a closure of the space $\mathcal{D}_{d}^{n}$, which we denote $\overline{\mathcal{D}_{d}^{n}}$. The 'discriminant' $\Sigma_{d}^{n}:=\overline{\mathcal{D}_{d}^{n}}-\mathcal{D}_{d}^{n}$ defines the singular discrete braid diagrams of period $d$.

Figure 1 summarizes the three types of braids introduced in this section.

2.4. Discretization: Back and forth. It is straightforward to pass from topological to discrete braids and back again.

Definition 2.3. Let $\mathbf{u} \in \Omega^{n}$ be a topological closed braid diagram. The period-d discretization of $\mathbf{u}$ is defined to be

$$
\operatorname{DISC}_{d}(\mathbf{u})=\left\{\operatorname{DISC}_{d}\left(u^{\alpha}\right)\right\}^{\alpha}:=\left\{u^{\alpha}(i / d)\right\}_{i}^{\alpha} .
$$

Conversely, given a discrete braid $\mathbf{u} \in \mathcal{D}_{d}^{n}$, we construct a piecewise-linear [PL] topological braid diagram, $\mathrm{PL}(\mathbf{u}):=\left\{\mathrm{PL}\left(u^{\alpha}\right)\right\}$, where $\mathrm{PL}\left(u^{\alpha}\right)$ is the $C^{0}$-strand given by

$$
\operatorname{PL}\left(u^{\alpha}\right)(x):=u_{\lfloor d \cdot x\rfloor}+(d \cdot x-\lfloor d \cdot x\rfloor)\left(u_{\lceil d \cdot x\rceil}-u_{\lfloor d \cdot x\rfloor}\right) .
$$

The following lemma is left as an exercise.

Lemma 2.4. Let $\mathbf{u} \in \Omega^{n}$ and $\mathbf{v} \in \mathcal{D}_{d}^{n}$.

(1) PL sends the discrete braid class $[\mathbf{v}]$ to a well-defined topological braid class $\{\mathrm{PL}(\mathbf{v})\}$.

(2) For d sufficiently large, $\left\{\mathrm{PL}\left(\operatorname{DISC}_{d}(\mathbf{u})\right)\right\}=\{\mathbf{u}\}$.

The second part of this lemma accommodates the obvious fact that braiding data is lost if the discretization is too coarse. This leads to the following definition:

Definition 2.5. A discretization period $d$ is admissible for $\mathbf{u} \in \Omega^{n}$ if

$$
\left\{\operatorname{PL}\left(\operatorname{DisC}_{d}(\mathbf{u})\right)\right\}=\{\mathbf{u}\} .
$$




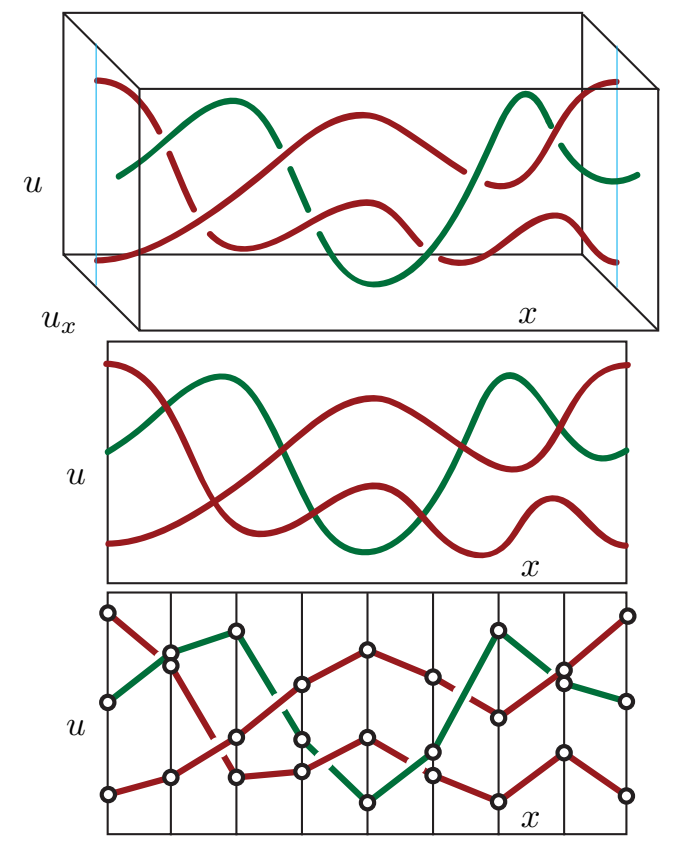

Figure 1. Three types of braids: a Legendrian topological braid [top], its braid diagram [center], and a discrete braid diagram [bottom].

In the next section, we will describe a Morse-Conley topological index for pairs of braids which relies on the algebraic length of the braid as a Morse function. Rather than detail the algebraic structures, we use an equivalent geometric formulation of length:

Definition 2.6. The length of a topological braid $\mathbf{u} \in \Omega^{n}$, denoted $\iota(\mathbf{u})$, is defined to be the total number of intersections in the braid diagram. If $\mathbf{u} \in \mathcal{D}_{d}^{n}$ is a discrete braid, then $\iota(\mathbf{u}):=\iota(\operatorname{PL}(\mathbf{u}))$.

\section{BRAID INVARIANTS}

We give a concise description of the invariant of [22] for relative discrete closed braids.

3.1. Relative braids. The motivation for the homotopy braid index is a forcing theory: given a stationary braid $\mathbf{v}$, does it force some other braid $\mathbf{u}$ to also be stationary with respect to the dynamics? This necessitates understanding how the strands of $\mathbf{u}$ braid relative to those of $\mathbf{v}$.

Definition 3.1. Given $\mathbf{v} \in \Omega^{m}$, define

$$
\Omega^{n} \text { REL } \mathbf{v}:=\left\{\mathbf{u} \in \Omega^{n}: \mathbf{u} \cup \mathbf{v} \in \Omega^{n+m}\right\} .
$$

The path components of $\Omega^{n}$ REL $\mathbf{v}$ comprise the relative braid classes, denoted $\{\mathbf{u}$ REL $\mathbf{v}\}$. In this setting, the braid $\mathbf{v}$ is called the skeleton. 
This procedure partitions $\Omega^{n}$ relative to $\mathbf{v}$ : not only are tangencies between strands of $\mathbf{u}$ illegal, so are tangencies with the strands of $\mathbf{v}$.

The definitions for discrete relative braids are analogous.

Definition 3.2. Given $\mathbf{v} \in \mathcal{D}_{d}^{m}$, define

$$
\mathcal{D}_{d}^{n} \text { REL } \mathbf{v}:=\left\{\mathbf{u} \in \mathcal{D}_{d}^{n}: \mathbf{u} \cup \mathbf{v} \in \mathcal{D}_{d}^{n+m}\right\} .
$$

The path components of $\mathcal{D}_{d}^{n}$ REL $\mathbf{v}$ comprise the relative discrete braid classes, denoted $[\mathbf{u}$ REL $\mathbf{v}]$. In this setting, the braid $\mathbf{v}$ is called the skeleton.

The operations $\operatorname{DISC}_{d}$ and PL have obvious extensions to relative braids by acting on both $\mathbf{u}$ and $\mathbf{v}$.

\subsection{Bounded and proper relative braids.}

Definition 3.3. A relative braid class $\{\mathbf{u}$ REL $\mathbf{v}\}$ is called proper if it is impossible to find an isotopy $\mathbf{u}(t)$ REL $\mathbf{v}$ such that $\mathbf{u}(0)=\mathbf{u}, \mathbf{u}(t)$ REL $\mathbf{v} \in\{\mathbf{u}$ REL $\mathbf{v}\}$, for $t \in[0,1)$, and $\mathbf{u}(1) \cup \mathbf{v} \in \Sigma^{n+m}$ is a diagram where an entire component of the braid $\mathbf{u}(1)$ has collapsed onto itself, another component of $\mathbf{u}(1)$, or a component of $\mathbf{v}$. A discrete relative braid class is proper if it is the discretization of a proper topological relative braid class.

The index we define is based on the topology of a relative braid class. It is most convenient to define this on compact spaces, hence the following definition.

Definition 3.4. A braid class (topological or discrete) is bounded if $\{\mathbf{u}$ REL $\mathbf{v}\}$ is a bounded set in $\overline{\Omega^{n}}$. (See Figure 21)
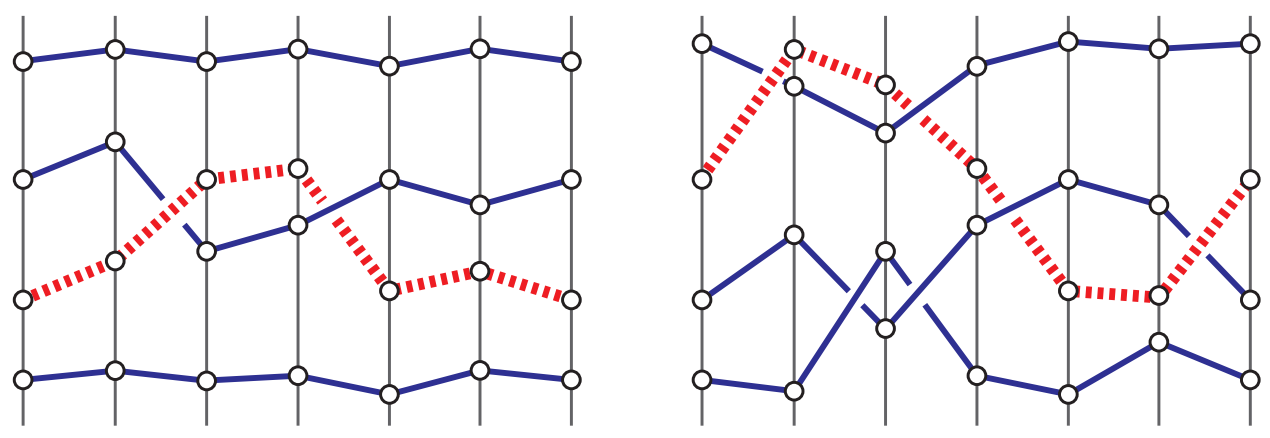

FiguRE 2. [left] a bounded but improper braid class; [right] a proper, but unbounded braid class. Solid strands are fixed; dashed strands are free.

For the remainder of the paper, all braids will be assumed proper and bounded unless otherwise stated.

3.3. The Conley index for braids. Consider a discrete relative braid class $[\mathbf{u}$ REL $\mathbf{v}] \subset \mathcal{D}_{d}^{n}$ which is bounded and proper. We associate to this class a Conleytype index for a class of dynamics on spaces of discrete braids. This will become an invariant of topological braids via discretization.

Denote by $N$ the closure of $[\mathbf{u}$ REL $\mathbf{v}]$ in the space $\overline{\mathcal{D}_{d}^{n}}$ REL $\mathbf{v}$. We identify an "exit set" on the boundary of $N$ consisting of those relative braids whose length 
$\iota$ can be decreased by a small perturbation. Let $\mathbf{w} \in \partial N$ denote a singular braid on the boundary of $N$ and let $W$ be a sufficiently small neighborhood of $\mathbf{w}$ in $\overline{\mathcal{D}_{d}^{n}}$ REL $\mathbf{v}$. Then $W$ is sliced by $\Sigma_{d}^{n}$ REL $\mathbf{v}$ into a finite number of connected components representing distinct neighboring braid classes, each component having a well-defined braid length $\iota \in \mathbb{Z}^{+}$. Define the exit set, $N^{-}$, of $N$ to be those singular braids at which $\iota$ can decrease:

$$
N^{-}:=\mathrm{CL}\{\mathbf{w} \in \partial N: \iota \text { is locally maximal on } \operatorname{INT}(N)\},
$$

where CL denotes closure in $\partial N$.

Definition 3.5. The Conley index of a discrete (proper, bounded, relative) braid class $[\mathbf{u}$ REL $\mathbf{v}]$ is defined to be the pointed homotopy class of spaces

$$
h([\mathbf{u} \text { REL v }])=\left[N / N^{-}\right]:=\left(N / N^{-},\left[N^{-}\right]\right) .
$$

Example 3.6. Consider the period-2 braid illustrated in Figure 3 [left] possessing exactly one free strand with anchor points $u_{0}$ and $u_{1}$. The anchor point in the middle, $u_{1}$, is free to move vertically between the fixed points on the skeleton. At the endpoints, one has a singular braid in $\Sigma$ which is on the exit set since a slight perturbation sends this singular braid to a different braid class with fewer crossings. The end anchor point, $u_{0}$, can move vertically between the two fixed points on the skeleton. The singular boundaries are in this case not on the exit set since pushing $u_{0}$ across the skeleton increases the number of crossings.
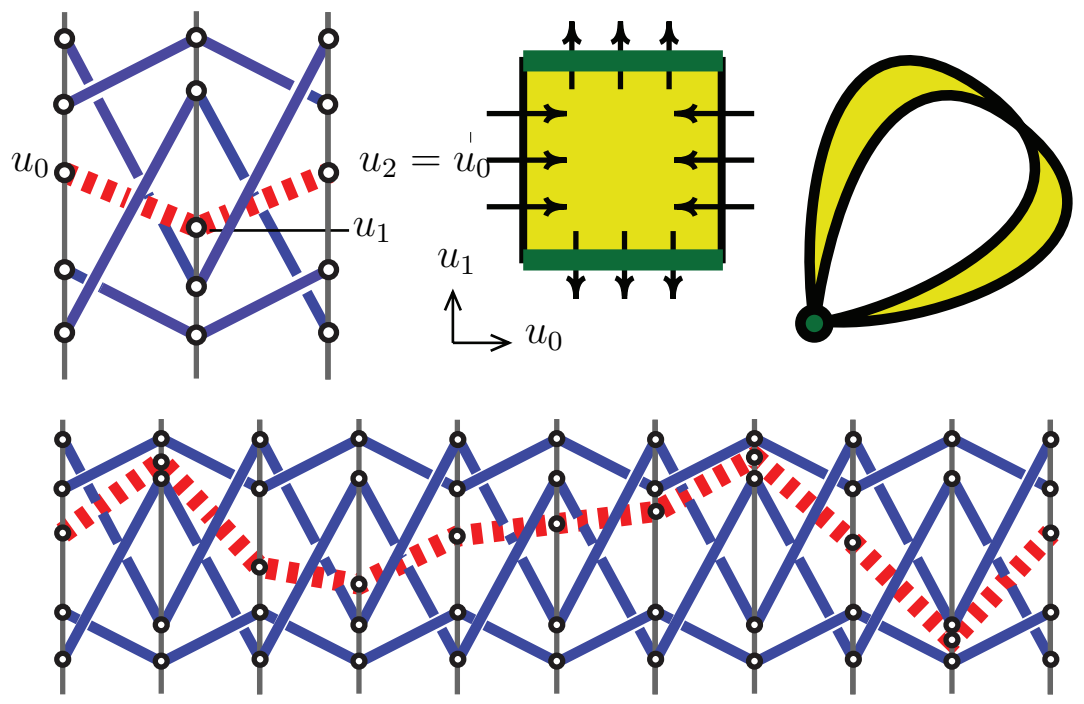

Figure 3. A period two braid [top, left] has configuration space a square [top, center]. Quotienting out the exit set leads to a space with homotopy type $S^{1}$ [top, right]. More examples can be obtained by lifting the skeleton along the spatial periodic variable: a period five generalization [bottom] has index $S^{2}$.

Since the points $u_{0}$ and $u_{1}$ can be moved independently, the configuration space $N$ in this case is the product of two compact intervals. The exit set $N^{-}$consists of 
those points on $\partial N$ for which $u_{1}$ is a boundary point. Thus, the homotopy index of this relative braid is $\left[N / N^{-}\right] \simeq S^{1}$.

By taking a chain of copies of this skeleton (i.e., taking a cover of the spatial domain), one can construct examples with one free strand weaving in and out of the fixed strands in such a way as to produce an index with homotopy type $S^{k}$ for any $k \geq 0$.

The extension of the Conley index to topological braid diagrams is straightforward: choose an admissible discretization period $d$, take the Conley index of the period- $d$ discretization, then show that this is independent of $d$. The key step (independence with respect to $d$ ) is, unfortunately, not true. For $d$ sufficiently small, there may be different discrete braid classes which define the same topological braid. The information from any one of these coarse components is incomplete. The following theorem, which is the main result from [22], resolves this obstruction.

Theorem 3.7 (see 22], Thm. 19 and Prop. 27). For d sufficiently large 2 the

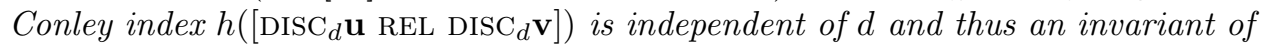
the topological braid class $\{\mathbf{u}$ REL $\mathbf{v}\}$.

Definition 3.8. Given a topological braid class $\{\mathbf{u}$ REL $\mathbf{v}\}$, define the homotopy index to be

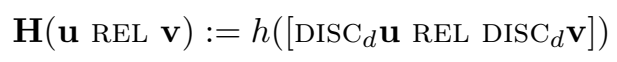

for $d$ sufficiently large.

For purposes of this paper, the homotopy index is defined with $d$ sufficiently large. This is well-defined, but not optimal for doing computations. To that end, one can use the more refined formula of 22, which computes $\mathbf{H}$ for any admissible discretization period $d$ via wedge sums: we will not require this complication in this paper.

For most applications it suffices to use the homological information of the index given by its Poincaré polynomial

$$
P_{\tau}(\mathbf{H}):=\sum_{k=0}^{\infty} \operatorname{dim} H_{k}(\mathbf{H}) \tau^{k}=\sum_{k=0}^{\infty} \operatorname{dim} H_{k}\left(N, N^{-}\right) \tau^{k} .
$$

This also has the pleasant corollary of making the index computable via rigorous homology algorithms.

\section{Dynamics AND the BRAid IndeX}

The homotopy braid invariant is defined as a "Conley index." This index has significant dynamical content.

The most basic version of the Conley index has the following ingredients [13]: given a continuous flow on a metric space, a subset $N$ is said to be an isolating block if all points on $\partial N$ leave $N$ under the flow in forwards and/or backwards time. The Conley index of $N$ with respect to the flow is then the pointed homotopy class $\left[N / N^{-}\right]$, where $N^{-}$denotes the exit set, or points on $\partial N$ which leave $N$ under the flow in forwards time. Standard facts about the index include (1) invariance of the index under continuous changes of the flow and the isolating block; and (2) the forcing result: a nonzero index implies that the flow has an invariant set in

\footnotetext{
${ }^{2}$ A sufficient though high lower bound is the number of crossings of $\mathbf{u}$ with itself and with $\mathbf{v}$.
} 
the interior of $N$. In order to implement Conley index theory in combination with braids we define the following class of dynamical systems.

Definition 4.1. Given $d>0$, a parabolic recurrence relation $\mathcal{R}$ on $\mathbb{Z} / d \mathbb{Z}$ is a collection of $C^{1}$-functions $\mathcal{R}_{i}: \mathbb{R}^{3} \rightarrow \mathbb{R}, i \in \mathbb{Z} / d \mathbb{Z}$ such that for each $i, \partial_{1} \mathcal{R}_{i}>0$ and $\partial_{3} \mathcal{R}_{i} \geq 0$. We say that $\mathcal{R}$ is exact if there exists a sequence of $C^{2}$-generating functions $S_{i}$ such that

$$
\mathcal{R}_{i}\left(u_{i-1}, u_{i}, u_{i+1}\right)=\partial_{2} S_{i-1}\left(u_{i-1}, u_{i}\right)+\partial_{1} S_{i}\left(u_{i}, u_{i+1}\right) \quad \forall i .
$$

A parabolic recurrence relation defines a vector field on $\overline{\mathcal{D}_{d}^{n}}$,

$$
\frac{d}{d t}\left(u_{i}^{\alpha}\right)=\mathcal{R}_{i}\left(u_{i-1}^{\alpha}, u_{i}^{\alpha}, u_{i+1}^{\alpha}\right),
$$

with all subscript operations interpreted modulo the permutation $\tau: u_{d+1}^{\alpha}=u_{1}^{\tau(\alpha)}$. The flow generated by Eqn. (4.2) is called a parabolic flow on $\mathcal{D}_{d}^{n}$. For more details, see [22. Exact parabolic recurrence relations induce a flow which is the gradient flow of $W(\mathbf{u}):=\sum_{i} S_{i}\left(u_{i}^{\alpha}, u_{i+1}^{\alpha}\right)$.

A parabolic flow acts on discrete braid diagrams in much the same way that Eqn. (1.1) acts on topological braid diagrams. As we have defined it in 3.3, the Conley index for a discrete braid class $[\mathbf{u}$ REL $\mathbf{v}]$ uses its closure $N=\operatorname{cl}[\mathbf{u}$ REL $\mathbf{v}]$ as an isolating block. Indeed, if [u REL $\mathbf{v}]$ is bounded, then $N$ is a compact set. If $\left[\mathbf{u}\right.$ REL v] is proper, then the vector field on $\overline{\mathcal{D}_{d}^{n}}$ REL $\mathbf{v}$ induced by $\mathcal{R}$ is transverse to $\partial N$, and $N$ is really an isolating block for the parabolic flow. The set $N^{-}$defined in the previous section then is the exit for $N$. This particular link lies at the heart of the theory and follows from a discrete version of the comparison principle 20, 26, 31. Details of the construction can be found in [22, where it is shown that the index $h([\mathbf{u}$ REL $\mathbf{v}])$ defined via Eqns. (3.1) and (3.2) is the Conley index of any parabolic recurrence relation which fixes $\mathbf{v}$. Figure 4 illustrates the action of a parabolic flow on braids.

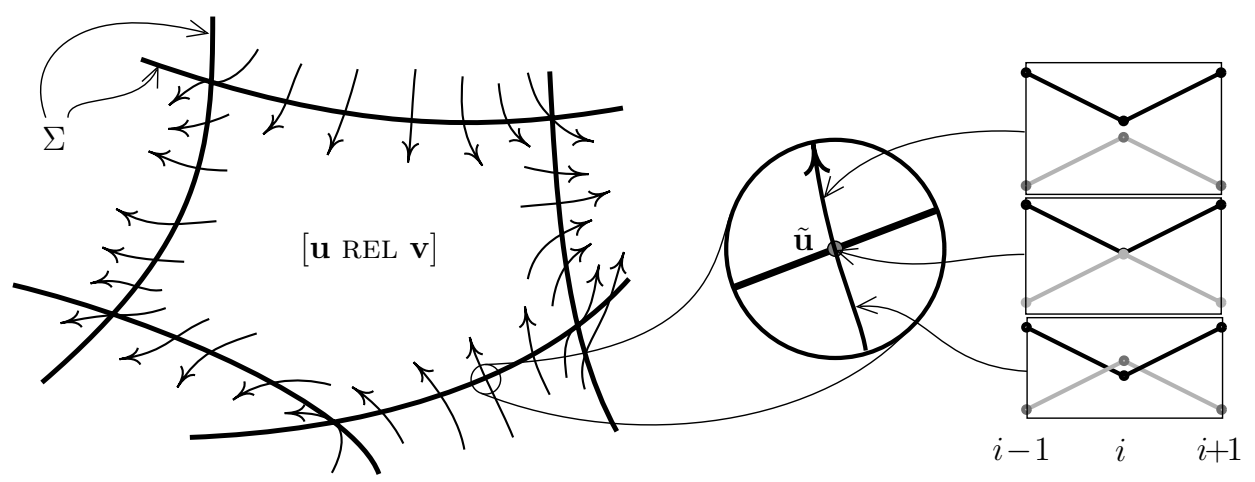

Figure 4. A parabolic flow on a (bounded and proper) braid class is transverse to the boundary faces, making the braid class into an isolating block. The local linking of strands decreases strictly along the flow lines at a singular braid $\tilde{\mathbf{u}}$.

In 22 it furthermore is shown that certain Morse inequalities hold for stationary solutions of Eqn. (4.2). The Morse inequalities also provide information about the 
periodic orbits. This is due to the fact that for parabolic systems the set of bounded solutions consists only of stationary points, periodic orbits, and connections between them.

Theorem 1.1 is an extension of the following results for parabolic lattice systems.

Theorem 4.2 (22). Let $\mathcal{R}$ be a parabolic recurrence relation. The induced flow on a bounded proper discrete braid class $[\mathbf{u}$ REL $\mathbf{v}]$, where $\mathbf{v}$ is a stationary skeleton, has an invariant solution within the class $[\mathbf{u}$ REL $\mathbf{v}]$ if the Conley index $h=h([\mathbf{u}$ REL v $])$ is nonzero. Furthermore:

(1) If the Euler characteristic $\chi(h) \neq 0$, then there exist stationary solutions of the braid class $[\mathbf{u}$ REL $\mathbf{v}]$.

(2) If $\mathcal{R}$ is exact, then the number of stationary solutions of braid class $[\mathbf{u}$ REL $\mathbf{v}]$ is bounded below by $\left|P_{\tau}(h)\right|$, the number of nonzero monomials of the Poincaré polynomial of the index.

If a proper bounded braid class [ $\mathbf{u}$ REL $\mathbf{v}$ ] contains no stationary braids for a particular recurrence relation $\mathcal{R}$, then $h(\mathbf{u}$ REL $\mathbf{v}) \neq 0$ forces periodic solutions of Eqn. (4.2), i.e. the components of $\mathbf{u}$ are periodic. If the system is nondegenerate, then the number of orbits is given by $P_{1}(h) / 2$. As a consequence in this case, $P_{\tau}(h)$ is divisible by $1+\tau$ and $\mathcal{R}$ is not exact. Note that for $d$ large enough the topological information is contained in the invariant $\mathbf{H}$ for the topological braid class $\{\mathbf{u}$ REL $\mathbf{v}\}$.

\section{Examples: Stationary SOlutions}

The following examples all satisfy hypotheses (g1) and (g2).

Example 5.1. Consider the following family of spatially inhomogeneous AllenCahn equations studied by Nakashima [28, 29]:

$$
\epsilon^{2} u_{t}=\epsilon^{2} u_{x x}+h(x) u\left(1-u^{2}\right)
$$

where $h: S^{1} \rightarrow(0,1)$ is not a constant. Clearly this equation has stationary solutions $u=0, \pm 1$ and is exact with Lagrangian

$$
L=\frac{1}{2} \epsilon^{2} u_{x}^{2}-\frac{1}{4} h(x) u^{2}\left(2-u^{2}\right) .
$$

According to [28, for any $N>0$, there exists an $\epsilon_{N}>0$ so that for all $0<$ $\epsilon<\epsilon_{N}$, there exist at least two stationary solutions which intersect $u=0$ exactly $N$ times. (The cited works impose Neumann boundary conditions: it is a simple generalization to periodic boundary conditions.)

Via Theorem 5.4 below we have that for any such $h$ and any small $\epsilon$, this equation admits an infinite collection of stationary periodic curves; furthermore, there is a lower bound of $N$ on the number of 1-periodic solutions.

Example 5.2. Consider the following equation:

$$
u_{t}=u_{x x}-\frac{5}{8} \sin 2 x u_{x}+\frac{\cos x}{\cos x+\frac{3}{\sqrt{5}}} u\left(u^{2}-1\right),
$$

with $x \in S^{1}=\mathbb{R} / 2 \pi \mathbb{Z}$.

Equation (1.4) is a weighted exact system with Lagrangian

$$
L=e^{-\frac{5}{16} \cos 2 x}\left(\frac{1}{2} u_{x}^{2}-\frac{\cos x}{\cos x+\frac{3}{\sqrt{5}}} \frac{\left(u^{2}-1\right)^{2}}{4}\right),
$$


where by "weighted exact" we mean (cf. Eqn. (1.2))

$$
u_{t}=e^{\frac{5}{16} \cos 2 x}\left[\frac{d}{d x} \frac{\partial L}{\partial u_{x}}-\frac{\partial L}{\partial u}\right] .
$$

One checks easily that there are stationary solutions $u= \pm 1$ and $u_{ \pm}=$ $\pm \frac{1}{2}(\sqrt{5} \cos x+1)$, as in Figure 5. These curves comprise a skeleton $\mathbf{v}=$ $\left\{-1, u_{-}, u_{+},+1\right\}$, which can be discretized to yield the skeleton of Example 3.6. From the computation of the index there, this skeleton forces a stationary solution of the braid class indicated in Figure 3 [left]: of course, this is detecting the obvious stationary solution $u=0$.

What is more interesting is the fact that one can take periodic extensions of the skeleton and add free strands in a manner which makes the relative braid spatially nonperiodic. Let us describe a family of proper and bounded relative braid classes. Let $\mathbf{v}^{n}$ be the $n$-fold periodic extension of $\mathbf{v}$ on $[0, n]$ and consider a single free strand $-1<u(x)<1$ that links with $\mathbf{v}^{n}$ as follows: on each interval $[k, k+1]$, $k=0, \ldots, n-1$, we choose one of three possibilities:

(a) $\iota\left(u, u_{-}\right)=0$ and $\iota\left(u, u_{+}\right)=2$,

(b) $\iota\left(u, u_{-}\right)=2$ and $\iota\left(u, u_{+}\right)=2$, or

(c) $\iota\left(u, u_{-}\right)=2$ and $\iota\left(u, u_{+}\right)=0$.

Define a symbol sequence $\sigma=\left(\sigma_{1} \ldots \sigma_{n}\right)$, where $\sigma_{i} \in\{a, b, c\}$. Every symbol sequence except for $\sigma=(a \ldots a)$ and $\sigma=(c \ldots c)$ defines a proper bounded braid class $\left\{\mathbf{u}_{\sigma}\right.$ REL $\left.\mathbf{v}\right\}$. Introduce the identification $\sigma \sim \sigma^{\prime}$ defined by $\sigma^{\prime}=\tau^{k}(\sigma)$, for some $0 \leq k \leq n-1$, where $\tau$ is a shift to the right. The equivalence classes are denoted by $[\sigma]$. It follows that if for two symbol sequences $\sigma$ and $\sigma^{\prime}$ it holds that $[\sigma] \neq\left[\sigma^{\prime}\right]$, then the associated braid classes $\left\{\mathbf{u}_{\sigma}\right.$ REL $\left.\mathbf{v}\right\}$ and $\left\{\mathbf{u}_{\sigma^{\prime}}\right.$ REL $\left.\mathbf{v}\right\}$ are different. This is easily seen when the total number of $a$ 's, b's or c's is different. In the case the number of symbols is the same no intersections can be created or are lost via a homotopy, since all intersections occur at isolated intervals contained in $[k, k+1]$, $0 \leq k \leq n-1$.

To compute the invariant, we discretize. Choose the discretization $d=2 n$ on $[0, n] 3$ Figure 3 [right] shows an example. In 93 the index was computed. Using the stabilization of the index as given by Theorem 3.7 we obtain:

$$
\mathbf{H}\left(\mathbf{u}_{\sigma} \text { REL } \mathbf{v}\right)=h\left(\operatorname{DISC}_{2 n} \mathbf{u}_{\sigma} \operatorname{REL~DISC}_{2 n} \mathbf{v}^{n}\right) \simeq S^{k}
$$

where $k=\#\{b \in \sigma\}$. Therefore, $P_{\tau}(\mathbf{H})=\tau^{k}$.

The Morse inequalities, in particular Theorem 1.1 and Theorem 1.2. now imply that for each $n>0$ there exist at least $3^{n}-2$ different stationary solutions. This information can be used again to prove that the time $2 \pi$-map of the stationary equation has positive entropy; see e.g. 30, 33.

Example 5.3. The following class of examples is very general and includes Example 5.1 as a special case. One says that Eqn. (1.1) is dissipative if

$$
u g(x, u, 0) \rightarrow-\infty \text { as }|u| \rightarrow+\infty
$$

uniformly in $x \in S^{1}$.

\footnotetext{
${ }^{3}$ This is admissible for the skeleton $\mathbf{v}^{n}$ and is large enough to yield the correct index computation.
} 


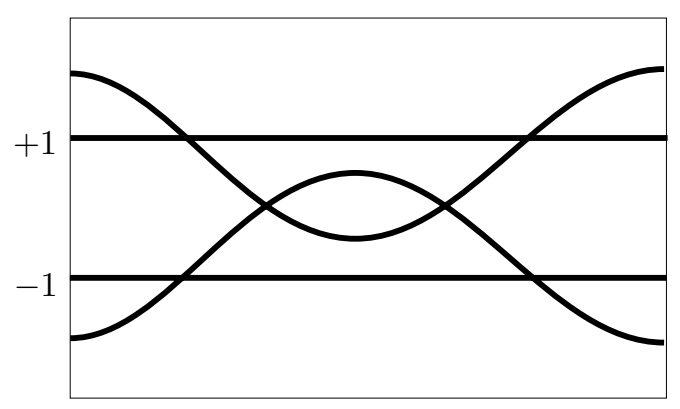

Figure 5. The skeleton of stationary solutions for Eqn. (1.4) forces an infinite collection of additional solutions which grows exponentially in the number of strands employed.

Theorem 5.4. Let $g$ be dissipative and satisfy $(\mathrm{g} 1)$ and $(\mathrm{g} 2)$. If $\mathbf{v}$ is a nontrivially braided stationary skeleton (i.e., $\iota(\mathbf{v}) \neq 0)$, then there are infinitely many braid classes represented as stationary solutions to Eqn. (1.1). Moreover, the number of braid classes for which $\mathbf{u}$ consists of just one strand is bounded from below by $\lceil\iota / 2\rceil-1$, where $\iota$ is the maximal number of intersections between two strands of $\mathbf{v}$.

Proof. Given the assumptions one can find $c_{+}>0$ and $c_{-}<0$ such that $\pm g\left(x, c_{ \pm}, 0\right)$ $>0$, and

$$
c_{-}<v^{\alpha}(x)<c_{+}
$$

for all strands $v^{\alpha}$ in $\mathbf{v}$. Using discrete enclosure via sub/super solutions, Lemma C.1 in Appendix C yields solutions $u_{+}$and $u_{-}$such that

$$
c_{-}<u_{-}(x)<v^{\alpha}(x)<u_{+}(x)<c_{+},
$$

for all $\alpha$. Assume without loss of generality that all strands in $\mathbf{v}$ are 1-periodic (if not, one can take an appropriate covering of $\mathbf{v}$ ). For the sake of convenience we may assume that $x \in S^{1} \equiv \mathbb{R} / \mathbb{Z}$. Select two intersecting strands which form the braid $\mathbf{w}=\left\{v^{\alpha_{1}}, v^{\alpha_{2}}\right\}$, and set $\iota(\mathbf{w})=\#\left\{\right.$ intersections between $v^{\alpha_{1}}$ and $\left.v^{\alpha_{2}}\right\}$. Consider the skeleton $\mathbf{z}=\left\{u_{-}, v^{\alpha_{1}}, v^{\alpha_{2}}, u_{+}\right\}$and a free strand $u(x)$ - with $u(x+1)=u(x)$ - that links with $\mathbf{z}$ as follows: (1) $u_{-}(x) \leq u(x) \leq u_{+}(x)$, (2) for some $k>0$, $\iota\left(u, v^{\alpha_{1}}\right)=\iota\left(u, v^{\alpha_{2}}\right)=2 k<\iota(\mathbf{w}) 4$ These two hypotheses describe the relative braid class $\{\mathbf{u}$ REL $\mathbf{z}\}$, which clearly is a proper and bounded class and therefore has a well-defined homotopy braid index $\mathbf{H}$. The index $\mathbf{H}$ is an invariant of the braid class and it can be computed for instance by studying a specific system of which all solutions are known.

Consider the equation $\epsilon^{2} u_{x x}+u-u^{3}=0$. If we choose $\epsilon=(\pi(\iota(\mathbf{w})+1))^{-1}$, then there exists a periodic solution $v_{1}(x)$ with period $T=2 / \iota(\mathbf{w})$. Define $v_{2}(x)=$ $v_{1}(x-(1 / \iota(\mathbf{w})))$; then if we consider $v_{1}$ and $v_{2}$ on the interval $[0,1]$, it follows that $\iota\left(v_{1}, v_{2}\right)=\iota(\mathbf{w})$. The skeleton $\mathbf{z}^{\prime}=\left\{-1, v_{1}, v_{2},+1\right\}$ is now topologically equivalent to $\mathbf{z}$. Moreover, the equation $\epsilon^{2} u_{x x}+u-u^{3}=0$ has a unique family of solutions $\{u(\cdot+\phi)\}$, which has the right linking properties with the skeleton $\mathbf{z}^{\prime}:-1<u(x)<$ 1 , and $\iota\left(u, v_{1}\right)=\iota\left(u, v_{2}\right)=\iota\left(u, v^{\alpha_{1}}\right)=\iota\left(u, v^{\alpha_{2}}\right)<\iota(\mathbf{w})$. Therefore, \{u REL $\left.\mathbf{z}^{\prime}\right\}$

\footnotetext{
${ }^{4}$ We assume without loss of generality that $\iota(\mathbf{w})>2 k \geq 2$. In the case that $\iota(\mathbf{w})=2$ or 3 , we consider coverings of $\mathbf{w}$.
} 
and $\{\mathbf{u}$ REL $\mathbf{z}\}$ are topologically equivalent. This particularly constructed equation with the skeleton $\mathbf{z}^{\prime}$ can now be used to compute $\mathbf{H}$.

Following the arguments in 4, 22, we can choose $\tau>0$ sufficiently small, i.e. $\frac{1}{\tau} \in$ $\mathbb{N}$, so that the time $\tau$-map of the equation, i.e. $(x, y) \mapsto\left(u(\tau ;(x, y)), u^{\prime}(\tau ;(x, y))\right)$, is a monotone (positive) twist map for $-1 \leq u \leq 1$; see also Appendix D. This allows us to apply the theory of parabolic recurrence relations in [22] with respect to the variables $u_{i}=u(\tau i)$, where $u$ is any solution of the equation $\epsilon^{2} u_{x x}+u-$ $u^{3}=0$ with $-1 \leq u \leq 1$. The discretized skeleton $\operatorname{DISC}_{d}\left(\mathbf{z}^{\prime}\right)$, with $d=\frac{1}{\tau}$, and $\operatorname{DISC}_{d}(\mathbf{u})$ REL DISC $d\left(\mathbf{z}^{\prime}\right)$ are of the same topological braid class as $\mathbf{z}^{\prime}$ and $\mathbf{u}$ REL $\mathbf{z}^{\prime}$ respectively. We can thus use Theorem 3.7 to compute $\mathbf{H}$.

The set of all critical points of the discretized system in $\left[\operatorname{DISC}_{d}(\mathbf{u}) \operatorname{REL} \operatorname{DISC}_{d}\left(\mathbf{z}^{\prime}\right)\right]$ is given by $\left\{\operatorname{DISC}_{d}(u(x+\phi)) \mid \phi \in \mathbb{R}\right\}$, which represents a hyperbolic circle of stationary strands. Its unstable manifold has dimension $\iota\left(u, v_{1}\right)=2 k$ and therefore its Morse polynomial is given by $\tau^{2 k-1}(1+\tau)$. Since this captures the entire invariant set, it follows from Theorem 3.7 that $P_{\tau}(h)=P_{\tau}(\mathbf{H})=\tau^{2 k-1}(1+\tau)$; see also [4] for details.

From the invariant $\mathbf{H}$ and Theorem 1.2 we deduce that if Eqn. (1.1) is dissipative and exact it has at least $\left\lceil\frac{\iota(\mathbf{w})}{2}\right\rceil-11$-periodic solutions. One finds infinitely many stationary braids by allowing periods $2 n$. Indeed, take the periodic extension $\mathbf{w}^{2 n}$. Then for any $k$ satisfying $2 k<\iota\left(\mathbf{w}^{2 n}\right)=2 n \iota(\mathbf{w})$ we find a $2 n$-periodic solution. By projecting this to the interval $[0,1]$ we obtain a multi-strand stationary braid for Eqn. (1.1). As a matter of fact for each pair $p, q$, with $q<p$ and $\operatorname{gcd}(p, q)=1$, there exists at least one solution $u_{p, q}$, by setting $k=\iota(\mathbf{w}) q$ and $n=p$. This infinity of solutions enshrouds the set $\mathbb{Q} \cap(0,1)$.

\section{ExAmples: Time-PERiodic SOlutions}

This is a longer example of a very general forcing result for time-periodic solutions.

Example 6.1. Consider equations of the following type as given by Eqn. (1.6):

$$
u_{t}=u_{x x}+u b(u)+u_{x} h\left(x, u, u_{x}\right), \quad x \in \mathbb{R} / \mathbb{Z},
$$

where the nonlinearity is assumed to satisfy (g1), i.e. $h$ has sublinear growth in $u_{x}$ at infinity. Moreover, assume that $b$ and $h$ satisfy the hypotheses:

(b1) $b(0)>0$, and $b$ has at least one positive and one negative root;

(b2) $h(x, 0,0)=0$, and $h>0$ on $\left\{u u_{x} \neq 0\right\}$.

Theorem 6.2. Under the hypotheses above, Eqn. (1.6) possesses an infinite collection of time-periodic solutions all with different braid classes.

Proof. Consider first the perturbed equation,

$$
u_{t}=u_{x x}+u b(u)+\alpha_{\epsilon} u_{x} h\left(x, u, u_{x}\right),
$$

where $\alpha_{\epsilon}=0$ for $\sqrt{u^{2}+u_{x}^{2}} \in[0, \epsilon]$ and $\alpha_{\epsilon}=1$ for $\sqrt{u^{2}+u_{x}^{2}} \geq 2 \epsilon$. For $\epsilon>0$ Eqn. (6.1) has small stationary solutions $v_{\epsilon}(x)$ which oscillate about $u=0$. We can choose this $v_{\epsilon}$ and a relatively prime pair of integers $p, q \in \mathbb{N}$ such that $v_{\epsilon}(x+p)=$ $v_{\epsilon}(x)$ and $\sqrt{b(0)} / 2 \pi \leq q / p$ is arbitrarily close to $q / p$. The integer $q$ represents the number of times the oscillation fits on the interval $[0, p]$.

We use (b1) to build a skeleton for Eqn. (6.1). Let $a_{+}$and $a_{-}$denote positive and negative roots of $b$, and consider the skeleton $\mathbf{v}=\left\{v^{1}, v^{2}, v^{3}, v^{4}\right\}$ on $\mathbb{R} / p \mathbb{Z}$ 
with $v^{1}(x)=a_{-}, v^{2}(x)=a_{+}, v^{3}(x)=v_{\epsilon}(x)$, and $v^{4}(x)=v_{\epsilon}(x-p / 2 q)$. Clearly $\iota\left(v^{3}, v^{4}\right)=2 q$. Define the relative braid class $\{\mathbf{u}$ REL $\mathbf{v}\}$ as follows: $\mathbf{u}=\{u\}$ is a (1-strand) braid satisfying $a_{-}<u(x)<a_{+}$and $\iota\left(u, v^{3}\right)=\iota\left(u, v^{4}\right)=2 r<2 q$. This braid class is proper and bounded, and its homotopy invariant $\mathbf{H}$ was computed in the previous section:

$$
P_{\tau}(\mathbf{H})=\tau^{2 r-1}(1+\tau) .
$$

We claim that for $0<\epsilon \ll 1$ there are no stationary solutions in $\{\mathbf{u}$ REL $\mathbf{v}\}$. Suppose that $u$ is stationary. One checks that the function

$$
H\left(u, u_{x}\right):=\frac{1}{2} u_{x}^{2}+u \int_{0}^{u} b(s) d s-\int_{0}^{u} \int_{0}^{s} b(r) d r d s
$$

has derivative

$$
\frac{d}{d x} H=-\alpha_{\epsilon} u_{x}^{2} h\left(x, u, u_{x}\right) .
$$

This term is nonpositive by (b2) and not identically zero by the fact that $u$ cannot be close to a constant (thanks to the intersection numbers). The periodic boundary condition leads to the desired contradiction.

Since $\mathbf{u}$ is a 1-strand braid it follows from Theorem 1.10 that $\{\mathbf{u}$ REL $\mathbf{v}\}$ contains a $t$-periodic solution $u_{\epsilon}(t, x)$ to Eqn. (6.1). By lifting the equation to the interval $[0, k p], k \in \mathbb{N}$, we obtain different periodic solutions for each $r<k q$, which shows that there are $t$-periodic solutions for infinitely many different braid classes; see [22, Lem. 43] for details. What remains is to show that these periodic solutions to Eqn. (6.1) persist in the limit $\epsilon \rightarrow 0$. We need to prove that the limits obtained are not equal to the zero solution. We use an argument similar to that of Angenent [4].

We wish to conclude that Eqn. (6.1) does not permit small bounded solutions as $\epsilon \rightarrow 0$ other than $u \equiv 0$. Let $x \in[0, p]$. Then linearization of Eqn. (6.1) about $u=0$ yields

$$
\psi_{t}=\psi_{x x}+b(0) \psi+R_{\epsilon}\left(x, \psi, \psi_{x}\right)
$$

where $\left|R_{\epsilon}\right| \leq C\left(|\psi|^{2}+\left|\psi_{x}\right|^{2}\right)$ for $|\psi|+\left|\psi_{x}\right|$ sufficiently small, uniformly in $x \in[0, p]$. The spectrum of the operator

$$
L=\frac{d^{2}}{d x^{2}}+b(0)
$$

on $L^{2}(\mathbb{R} / p \mathbb{Z})$ is given by the eigenvalues $\lambda_{n}=-4 \pi^{2} n^{2} / p^{2}+b(0)$, and associated eigenfunctions $\phi_{n}$, for $n=0,1, \ldots$. Since $\sqrt{b(0)} / 2 \pi \leq q / p$ it follows that $\lambda_{n}>0$ for all $n<q$, and $\lambda_{n} \leq 0$ for $n \geq q$. This yields a (spectral decomposition) splitting of $L=L_{+}+L_{-}$. Define the set $I_{r}=\{u \mid \iota(u, 0)=2 r<2 q\}$. Since $\iota\left(\phi_{n}, 0\right)=2 n$ we have that $\operatorname{ker} L_{+} \notin I_{r}$. A straightforward analysis then shows that for all $u \in \cos \left(I_{r}\right)$ it follows that $\left\|L_{+} u\right\|_{L^{2}} \geq K\|u\|_{L^{2}}$, for some $K>0$.

Define the function $B(\psi)=\frac{1}{2}\left(\psi, L_{+} \psi\right)_{L^{2}}$. Then any solution $\psi(t, \cdot)$ in $\{\mathbf{u}$ REL $\mathbf{v}\}$ close to $u=0$ in $L^{\infty}$, i.e. $|\psi(t, x)|<\delta \ll 1$ independent of $\epsilon$, satisfies $\left|\left(R_{\epsilon}, L_{+} \psi\right)_{L^{2}}\right|$ $\leq C\|\psi\|_{L^{2}}^{3}+C\left\|\psi_{x}\right\|_{L^{2}}^{2}\|\psi\|_{L^{2}}$. If we use the regularity result in Appendix B by keeping track of $|\psi|$ in the proof we obtain the estimate $\left|\psi_{x}\right| \leq C|\psi|$. Therefore,

$$
\begin{aligned}
\frac{d}{d t} B(\psi) & =\left(L_{+} \psi, L_{+} \psi\right)_{L^{2}}+\left(R_{\epsilon}\left(x, \psi, \psi_{x}\right), L_{+} \psi\right)_{L^{2}} \\
& \geq K\|\psi\|_{L^{2}}^{2}-C\|\psi\|_{L^{2}}^{3}-C\left\|\psi_{x}\right\|_{L^{2}}^{2}\|\psi\|_{L^{2}} \\
& \geq K\|\psi\|_{L^{2}}^{2}-C^{\prime}\|\psi\|_{L^{2}}^{3}>0 .
\end{aligned}
$$


Consequently, $B(\psi(t, x)) \leq B\left(\psi\left(t_{0}, x\right)\right)$ for $t \leq t_{0}$. Since $\partial_{t} B(\psi)>0$, it follows that $\|\psi(t, \cdot)\|_{L^{2}} \rightarrow 0$ as $t \rightarrow-\infty$, and thus $\psi(t, x) \rightarrow 0$ as $t \rightarrow-\infty$. This then contradicts the fact that $\psi(t, \cdot)$ is a small solution in $\{\mathbf{u}$ REL $\mathbf{v}\}$.

Remark 6.3. The form of Eqn. (1.6) is not the most general form possible. Certainly, having $h$ strictly negative on $\left\{u u_{x} \neq 0\right\}$ is also permissible. One should also be able to weaken the condition $h(x, 0,0)=0$. This requires a careful study of the linearized operator

$$
L=\frac{d^{2}}{d x^{2}}+h(x, 0,0) \frac{d}{d x}+b(0) .
$$

Crucial in this case is to be able to link sign changes of the eigenfunctions to $q$ and $p$; see also [5].

\section{Proofs: Forcing Stationary solutions}

7.1. Discretization of the equation. For the remainder of this section, we work with individual strands $u=u^{\alpha}$ in $\mathbf{u}=\left(u^{\alpha}\right)$, suppressing the superscripts for notational aesthetics.

We discretize Eqn. (1.1) in the standard manner. Choose a step size $1 / d$, for $d \in \mathbb{N}$, and define $u_{i}:=u(i / d)$. We approximate the first derivative $u_{x}(i / d)$ by $\Delta u_{i}:=d\left(u_{i+1}-u_{i}\right)$ and the second derivative $u_{x x}(i / d)$ by $\Delta^{2} u_{i}:=$ $d^{2}\left(u_{i+1}-2 u_{i}+u_{i-1}\right)$.

Lemma 7.1. Let $\mathbf{u}$ be a stationary braid for Eqn. (1.1). Then

$$
\left.\epsilon_{i}(d):=\Delta^{2} u_{i}+g\left(\frac{i}{d}, u_{i}, \Delta u_{i}\right)\right) \longrightarrow 0,
$$

as $d \rightarrow \infty$ uniformly in $i$. In particular, $\left|\epsilon_{i}(d)\right| \leq C / d$.

Proof. From Appendix A it follows that each strand $u$ of a stationary solution to Eqn. (1.1) is $C^{3}$. A Taylor expansion yields

$$
\begin{aligned}
\Delta u_{i}-u_{x} & =d \cdot\left(u((i+1) / d)-u_{i}(i / d)\right)-u_{x}(i / d) \\
& =d \cdot R_{1 / d}^{2}(i / d)=\frac{1}{2} u_{x x}(y) / d, \text { for some } y, \\
\Delta^{2} u_{i}-u_{x x} & =d^{2} \cdot\left(u((i+1) / d)-2 u_{i}(i / d)+u((i-1) / d)\right)-u_{x x}(i / d) \\
& =d^{2} \cdot R_{1 / d}^{3}(i / d)=\frac{1}{6} u_{x x x}(y) / d . \text { for some } y .
\end{aligned}
$$

For $x_{0}:=i / d$ it therefore holds that

$$
\left|\Delta u_{d \cdot x_{0}}-u_{x}\left(x_{0}\right)\right| \leq C / d, \quad\left|\Delta^{2} u_{d \cdot x_{0}}-u_{x x}\left(x_{0}\right)\right| \leq C / d,
$$

with $C$ independent of $x_{0}$. Since $f$ is $C^{1}$ the desired result follows. A more detailed asymptotic expansion for $\epsilon_{i}$ is obtained as follows:

$$
\begin{aligned}
\epsilon_{i}(d)= & u_{x x}(i / d)-\Delta^{2} u_{i}+g\left(i / d, u_{i}, \Delta u_{i}\right)-g\left(i / d, u(i / d), u_{x}(i / d)\right) \\
= & \partial_{u_{x}} g\left(i / d, u(i / d), u_{x}(i / d)\right)\left[\Delta u_{i}-u_{x}(i / d)\right] \\
& +\left[\Delta^{2} u_{i}-u_{x x}(i / d)\right]+R_{2} .
\end{aligned}
$$

From the weak form of Taylor's Theorem the remainder term $R_{2}$ satisfies $\left|R_{2}\right|=$ $o(1 / d)$. Combining this with the estimates obtained above we derive that $\left|\epsilon_{i}(d)\right| \leq$ $C / d$, thus completing the proof. 
The next step is to ensure that the $d$-point discretization of $\mathbf{u}$ is in fact a solution of an appropriate parabolic recurrence relation.

Lemma 7.2. Let $\mathbf{u} \in \Omega^{n}$, and let $d$ be an admissible discretization for $\mathbf{u}$. Then for any sequence $\left\{\epsilon_{i}^{\alpha}\right\}$ with $i=0, \ldots, d$ and $\alpha=1, \ldots, n$, there exists a parabolic recurrence relation $\mathcal{E}_{i}^{d}$ satisfying

$$
\mathcal{E}_{i}^{d}\left(u_{i-1}^{\alpha}, u_{i}^{\alpha}, u_{i+1}^{\alpha}\right)=-\epsilon_{i}^{\alpha},
$$

where $u_{i}^{\alpha}=u^{\alpha}(i / d)$. In addition, $\left|\mathcal{E}_{i}^{d}(r, s, t)\right| \leq C \max _{i, \alpha}\left|\epsilon_{i}^{\alpha}\right|$ for all $|r|,|s|,|t| \leq$ $2 \max _{i, \alpha}\left|u_{i}^{\alpha}\right|$ and some uniform constant $C$ depending only on $\mathbf{u}$.

Proof. This proof is a straightforward extension of [22, Lemma 55], in which $\epsilon_{i}^{\alpha} \equiv$ 0 .

Let $\mathbf{v} \in \Omega^{m}$ be stationary for Eqn. (1.1) and let $\{\mathbf{u}$ REL $\mathbf{v}\}$ be a bounded proper braid class with $d$ a sufficiently large discretization period. We now construct a parabolic recurrence relation for which the discrete skeleton $\operatorname{DISC}_{d} \mathbf{v}$ is stationary. Combining Lemmas 7.1 and 7.2 , the recurrence relation defined by

$$
\mathcal{R}_{i}^{d}\left(u_{i-1}, u_{i}, u_{i+1}\right):=\Delta^{2} u_{i}+g\left(i / d, u_{i}, \Delta u_{i}\right)+\mathcal{E}_{i}^{d}\left(u_{i-1}, u_{i}, u_{i+1}\right)
$$

has DISC $_{d} \mathbf{v}$ as a stationary solution. The above construction works for any $d^{\prime} \geq d$. For a given $d$ the recurrence relation $\mathcal{R}_{i}^{d}$ is considered on the compact set cl[ $[\mathbf{u}$ REL $\mathbf{v}]$, which implies that $\left|u_{i}\right|<2 \max _{i, \alpha}\left|v_{i}\right|$. To verify the parabolicity of $\mathcal{R}_{i}^{d}$, we compute the derivatives. From the parabolicity of $\mathcal{E}_{i}^{d}$, we obtain

$$
\partial_{1} \mathcal{R}_{i}^{d}=d^{2}+\partial_{1} \mathcal{E}_{i}^{d} \geq \lambda \cdot d^{2}>0 \text {. }
$$

Furthermore,

$$
\partial_{3} \mathcal{R}_{i}^{d}=d^{2}+\frac{\partial g}{\partial u_{x}} \cdot d+\partial_{3} \mathcal{E}_{i}^{d},
$$

which does not yet prove parabolicity since no estimates for $\partial_{u_{x}} g$ are given. However, utilizing hypothesis (g1), we have that

$$
\begin{aligned}
\mathcal{R}_{i}^{d}\left(u_{i-1}, u_{i}, u_{i+1}\right) & =\Delta^{2} u_{i}+g\left(i / d, u_{i}, \Delta u_{i}\right)+\mathcal{E}_{i}^{d}\left(u_{i-1}, u_{i}, u_{i+1}\right) \\
& \geq-C-C\left|\Delta u_{i}\right|^{\gamma}+\Delta^{2} u_{i} \\
& \geq-C-C d^{\gamma}+d^{2}\left(u_{i+1}-2 u_{i}+u_{i-1}\right),
\end{aligned}
$$

which shows that $\mathcal{R}_{i}^{d}$ is an increasing function of $u_{i+1}$, provided that $d$ is large enough. This relies on the fact that the braid class is bounded. Periodicity of $f$ implies that $R_{i+d}^{d}=\mathcal{R}_{i}^{d}$. Initially $\mathcal{R}_{i}^{d}(r, s, t)$ is defined for $|r|,|s|,|t| \leq 2 \max _{i, \alpha}\left|v_{i}\right|$. It is clear that $\mathcal{R}_{i}^{d}$ can easily be extended to a parabolic recurrence relation on all of $\mathbb{R}^{3}$.

7.2. Convergence to a stationary solution. Choose $d_{*}$ large enough such that $\mathcal{R}_{i}^{d}$ is parabolic for all $d \geq d_{*}$. Let $\left\{u_{i}^{\alpha, d}\right\}$ be a sequence of braids which are solutions of

$$
\mathcal{R}_{i}^{d}\left(u_{i-1}, u_{i}, u_{i+1}\right)=\Delta^{2} u_{i}+g\left(i / d, u_{i}, \Delta u_{i}\right)+\mathcal{E}_{i}^{d}\left(u_{i-1}, u_{i}, u_{i+1}\right)=0,
$$

and which satisfy the uniform estimate $\left|u_{i}^{\alpha, d}\right| \leq C$ for all $d$. For notational simplicity, we omit the discretization period $d$ and write $u_{i}$ instead of $u_{i}^{\alpha, d}$ in what follows. The discretization index will be clear from the range of the index $i$. 
Lemma 7.3. Let $\left\{u_{i}\right\}$ satisfy $\mathcal{R}_{i}^{d}\left(u_{i-1}, u_{i}, u_{i+1}\right)=0$ and $\left|u_{i}\right| \leq C$ as $d \rightarrow \infty$. Then

$$
\sum_{i=0}^{d} \frac{1}{d}\left|u_{i}\right|^{2} \leq C, \quad \sum_{i=0}^{d} d \cdot\left|u_{i+1}-u_{i}\right|^{2} \leq C,
$$

with $C$ independent of $d$.

Proof. For each strand $\alpha$ it holds that either $u_{i+d}=u_{i}$, or $u_{i+k d}=u_{i}$ for some $k$. Since there are only finitely many strands, the constant $k$ can be chosen uniformly for all $\alpha$. Therefore we assume without loss of generality that the first equality holds. The first estimate immediately follows from the uniform bound on $u_{i}$.

From Eqn. (7.4) it follows that

$$
-\Delta^{2} u_{i} \cdot \frac{1}{d} u_{i}=g\left(i / d, u_{i}, \Delta u_{i}\right) \frac{1}{d} u_{i}+\mathcal{E}_{i}^{d}\left(u_{i-1}, u_{i}, u_{i+1}\right) \frac{1}{d} u_{i} .
$$

From the periodic boundary conditions we have

$$
-\sum_{i=0}^{d} \Delta^{2} u_{i} \cdot \frac{1}{d} u_{i}=\sum_{i=0}^{d} d \cdot\left|u_{i+1}-u_{i}\right|^{2} .
$$

Combining the above estimates and using (g1) we obtain

$$
\begin{aligned}
\sum_{i=0}^{d} d \cdot\left|u_{i+1}-u_{i}\right|^{2} & \leq \sum_{i=0}^{d}\left(\frac{1}{d}\left|g\left(i / d, u_{i}, \Delta u_{i}\right)\right|\left|u_{i}\right|+\frac{1}{d}\left|\mathcal{E}_{i}^{d}\right|\left|u_{i}\right|\right) \\
& \leq C / d+\sum_{i=0}^{d} \frac{1}{d}\left[C_{\epsilon}+\epsilon d^{2}\left|u_{i+1}-u_{i}\right|^{2}\right]\left|u_{i}\right| \\
& \leq C_{\epsilon}+\epsilon C \sum_{i=0}^{d} d \cdot\left|u_{i+1}-u_{i}\right|^{2}, \quad \text { for any } \epsilon>0 .
\end{aligned}
$$

Choosing $\epsilon$ small enough yields the second estimate.

Define $\phi_{d}:=\operatorname{PL}\left(\left\{u_{i}\right\}\right)$. Then $\left\|\phi_{d}\right\|_{L^{2}}^{2} \leq \sum_{i=0}^{d} \frac{1}{d}\left|u_{i}\right|^{2}$ and $\left\|\frac{d}{d x} \phi_{d}\right\|_{L^{2}}^{2}=\sum_{i=0}^{d} d$. $\left|u_{i+1}-u_{i}\right|^{2}$. Due to the uniform estimates we obtain the Sobolev bound $\left\|\phi_{d}\right\|_{H^{1,2}} \leq$ $C$, with $C$ independent of $d$. Therefore, $\phi_{d_{n}}$ converges to some function $u \in$ $C^{0}([0,1])$, as $d_{n} \rightarrow \infty$.

Lemma 7.4. Let $\left\{u_{i}\right\}$ satisfy $\mathcal{R}_{i}^{d}\left(u_{i-1}, u_{i}, u_{i+1}\right)=0$ and $\left|u_{i}\right| \leq C$ as $d \rightarrow \infty$. Then

$$
\sum_{i=0}^{d} d \cdot\left|\Delta u_{i}-\Delta u_{i-1}\right|^{2 / \gamma} \leq C,
$$

with $C$ independent of $d$.

Proof. As in the proof of Lemma 7.3, we have

$$
\Delta^{2} u_{i}=-g\left(i / d, u_{i}, \Delta u_{i}\right)-\mathcal{E}_{i}^{d}\left(u_{i-1}, u_{i}, u_{i+1}\right) .
$$

Combining this estimate with (g1) we obtain

$$
d \cdot\left|\Delta u_{i}-\Delta u_{i-1}\right| \leq C+C\left|\Delta u_{i}\right|^{\gamma} .
$$


Therefore

$$
\begin{aligned}
\sum_{i=0}^{d} d^{\frac{2}{\gamma}-1}\left|\Delta u_{i}-\Delta u_{i-1}\right|^{2 / \gamma} & \leq C+C \sum_{i=0}^{d} \frac{1}{d}\left|\Delta u_{i}\right|^{2} \\
& \leq C \text { by Lemma 7.3 }
\end{aligned}
$$

which is the desired estimate.

Set $\psi_{d}:=\operatorname{PL}\left(\left\{\Delta u_{i}\right\}\right)$. Then $\left\|\psi_{d}\right\|_{L^{2}}^{2} \leq \sum_{i=0}^{d} \frac{1}{d}\left|\Delta u_{i}\right|^{2} \leq C$, and $\left\|\frac{d}{d x} \psi_{d}\right\|_{L^{2 / \gamma}}^{2}=$ $\sum_{i=0}^{d} d^{\frac{2}{\gamma}-1} \cdot\left|\Delta u_{i+1}-\Delta u_{i}\right|^{2 / \gamma} \leq C$. This implies that $\left\|\psi_{d}\right\|_{H^{1,2 / \gamma}} \leq C$, independent of $d$. Therefore there exists a subsequence $\psi_{d_{n}}$ converging to some function $v \in$ $C^{0}([0,1])$.

Lemma 7.5. Let $\left\{u_{i}\right\}$ satisfy $\mathcal{R}_{i}^{d}\left(u_{i-1}, u_{i}, u_{i+1}\right)=0$, and $\left|u_{i}\right| \leq C$ as $d \rightarrow \infty$. Then

$$
\left|\Delta u_{i}\right| \leq C, \quad\left|\Delta^{2} u_{i}\right| \leq C
$$

with $C$ independent of $d$.

Proof. The first estimate follows from the fact that $\left\|\psi_{d}\right\|_{C^{0}} \leq C$; hence $\left|\Delta u_{i}\right| \leq C$. For the second estimate we use Eqn. (7.7). The uniform bound on $\Delta u_{i}$ then yields a uniform bound on $\Delta^{2} u_{i}$.

Finally, we require an estimate on $\Delta^{3} u_{i}=d \cdot\left(\Delta^{2} u_{i+1}-\Delta^{2} u_{i}\right)$.

Lemma 7.6. Let $\left\{u_{i}\right\}$ satisfy $\mathcal{R}_{i}^{d}\left(u_{i-1}, u_{i}, u_{i+1}\right)=0$ and $\left|u_{i}\right| \leq C$ as $d \rightarrow \infty$. Then

$$
\left|\Delta^{3} u_{i}\right|=d \cdot\left|\Delta^{2} u_{i+1}-\Delta^{2} u_{i}\right| \leq C,
$$

with $C$ independent of $d$.

Proof. Since $\mathcal{R}_{i+1}^{d}-\mathcal{R}_{i}^{d}=0$ it follows from the definition of $\mathcal{R}_{i}^{d}$ that

$$
\begin{aligned}
\Delta^{2} u_{i+1}-\Delta^{2} u_{i} & +g\left((i+1) / d, u_{i+1}, \Delta u_{i+1}\right)-g\left(i / d, u_{i}, \Delta u_{i}\right) \\
& =-\mathcal{E}_{i+1}^{d}\left(u_{i}, u_{i+1}, u_{i+2}\right)+\mathcal{E}_{i}^{d}\left(u_{i-1}, u_{i}, u_{i+1}\right) .
\end{aligned}
$$

Using Taylor's theorem we obtain that

$$
\begin{aligned}
\partial_{x} g\left(i / d, u_{i}, \Delta u_{i}\right) \frac{1}{d} & +\partial_{u} g\left(i / d, u_{i}, \Delta u_{i}\right)\left(u_{i+1}-u_{i}\right) \\
& +\partial_{u_{x}} g\left(i / d, u_{i}, \Delta u_{i}\right)\left(\Delta u_{i+1}-\Delta u_{i}\right) \\
& +\left(\Delta^{2} u_{i+1}-\Delta^{2} u_{i}\right) \\
& =-\left(\mathcal{E}_{i+1}^{d}-\mathcal{E}_{i}^{d}\right)-R_{2}\left(i / d, u_{i}, \Delta u_{i}, \Delta^{2} u_{i}\right) .
\end{aligned}
$$

For $\Delta^{3} u_{i}$ this implies

$$
\begin{aligned}
\Delta^{3} u_{i} & =-\partial_{x} g\left(i / d, u_{i}, \Delta u_{i}, \Delta^{2} u_{i}\right)-\partial_{u} f\left(i / d, u_{i}, \Delta u_{i}, \Delta^{2} u_{i}\right) \Delta u_{i} \\
& -\partial_{u_{x}} f\left(i / d, u_{i}, \Delta u_{i}, \Delta^{2} u_{i}\right) \Delta^{2} u_{i}-\Delta \mathcal{E}_{i}^{d}-R_{2}\left(i / d, u_{i}, \Delta u_{i}, \Delta^{2} u_{i}\right) d .
\end{aligned}
$$

By Lemma 7.5 the right hand side is uniformly bounded in $d$, which provides the desired estimate on $\Delta^{3} u_{i}$.

Define $\chi_{d}:=\operatorname{PL}\left(\left\{\Delta^{2} u_{i}\right\}\right)$. From Lemma[7.6 we then derive that $\left\|\frac{d}{d x} \chi_{d}\right\|_{L^{\infty}} \leq C$. Therefore $\chi_{d_{n}}$ converges to some limit function $w$ in $C^{0}([0,1])$.

From Lemmas 7.3. 7.4, and 77.6 it follows that the functions $\phi_{d}, \psi_{d}$ and $\chi_{d}$ converge to the functions $u, v$ and $w$, respectively, with the anchor points being solutions of $\mathcal{R}_{i}^{d}=0$.

The following lemma relates discretized braids to stationary braids in $\{\mathbf{u}$ REL $\mathbf{v}\}$. 
Lemma 7.7. Let $u, v, w \in C^{0}([0,1])$ and let $\left\{u_{i}^{d}\right\}_{i=0}^{d}$ be sequences whose PL interpolations satisfy

$$
\operatorname{PL}\left(u_{i}^{d}\right) \rightarrow u, \quad \operatorname{PL}\left(\Delta u_{i}^{d}\right) \rightarrow v, \quad \operatorname{PL}\left(\Delta^{2} u_{i}^{d}\right) \rightarrow w
$$

in $C^{0}([0,1])$ as $d \rightarrow \infty$. If $\mathcal{R}_{i}^{d}\left(u_{i-1}^{d}, u_{i}^{d}, u_{i+1}^{d}\right)=0$ and $\left|\Delta^{3} u_{i}\right| \leq C$, then $u \in$ $C^{2}([0,1]), u_{x}=v$, and $u_{x x}=w$ satisfying $u_{x x}+g\left(x, u, u_{x}\right)=0$ pointwise on $[0,1]$.

Proof. We start with the estimate $\left|\phi_{d}^{\prime}-\psi_{d}\right| \leq \frac{1}{d} \max _{0 \leq i \leq d}\left|\Delta^{2} u_{i}\right| \rightarrow 0$ uniformly as $d \rightarrow \infty$. This implies that $\psi_{d} \rightarrow v$ in $C^{0}([0,1])$. The same estimate holds for $\left|\psi_{d}^{\prime}-\chi_{d}\right| \leq \frac{1}{d} \max _{0 \leq i \leq d}\left|\Delta^{3} u_{i}\right| \rightarrow 0$ uniformly as $d \rightarrow \infty$. Hence we deduce that $\chi_{d} \rightarrow w$ in $C^{0}([0,1])$. From the definition of derivatives it now follows that $\left\|D_{1 / d} u-v\right\|_{L^{\infty}} \rightarrow 0$, and $\left\|D_{1 / d} v-w\right\|_{L^{\infty}} \rightarrow 0$; thus $v=u_{x}$ and $w=u_{x x}$. From Lemma 7.1 we deduce that $u_{x x}+g\left(x, u, u_{x}\right)=0$.

Note that Lemma A.1 in Appendix A implies further that $u \in C^{3}([0,1])$.

7.3. Proof of Theorem 1.1. Given $P_{-1} \mathbf{H} \neq 0$, the existence of a single stationary solution is argued as follows. Choose $d_{*}$ large enough. Then from Theorem 4.2 it follows that Eqn. (7.4) has a discrete braid solution $\left\{u_{i}^{\alpha, d}\right\}$ for all $d \geq d_{*}$. The boundedness of the braid class implies that the sequence $\left\{u_{i}^{\alpha, d}\right\}$ satisfies

$$
\left|u_{i}^{\alpha, d}\right| \leq C, \quad \forall i, \alpha, \text { and } \forall d \geq d_{*} .
$$

Lemmas 7.177.7 imply that as $d \rightarrow \infty$ one obtains a stationary braid $\mathbf{u}=\left\{u^{\alpha}\right\}$ whose strands $u^{\alpha}$ satisfy the equation $u_{x x}+g\left(x, u^{\alpha}, u_{x}^{\alpha}\right)=0$. Since the skeletal strands $\operatorname{DISC}_{d}\left(v^{\beta}\right)$ converge to $v^{\beta}$ by construction and the pairwise intersection numbers are the same for all $d$, we have in the limit a solution to Eqn. (1.1) in the correct braid class. In the exact case, the conclusion follows from the condition $P_{\tau}(\mathbf{H}) \neq 0$, proving Theorem 1.2 .

\section{Proofs: Forcing Periodic SOlutions}

In this section, we provide details of the forcing arguments in the case of nonstationary solutions. The technique is philosophically the same as for stationary solutions: discretize, apply the Morse-theoretic results of [22, then prove convergence to solutions of Eqn. (1.1). However, the requisite estimates are more involved in the time-periodic case. Appendix B details a regularity result for nonstationary solutions to Eqn. (1.1).

8.1. Discretization and convergence. We begin by truncating the system. Consider the equation

$$
u_{t}=u_{x x}+g_{K}\left(x, u, u_{x}\right),
$$

where

$$
g_{K}\left(x, u, u_{x}\right):=\left\{\begin{array}{cc}
g\left(x, u, u_{x}\right) & \text { for }|u|+\left|u_{x}\right| \leq K, \\
\inf _{|u|+\left|u_{x}\right| \geq K}\left|g\left(x, u, u_{x}\right)\right| & \text { for }|u|+\left|u_{x}\right| \geq K
\end{array}\right.
$$

Consequently,

$$
\left|g_{K}\left(x, u, u_{x}\right)\right| \leq\left|g\left(x, u, u_{x}\right)\right|
$$

for all $x \in S^{1}, u, u_{x} \in \mathbb{R}$. Thanks to this, the estimates from Appendix B hold with the same constants: any complete uniformly bounded solution $u^{K}(t, x)$ to Eqn. (8.1) satisfies

$$
\left|u_{x}^{K}\right|+\left|u_{x x}^{K}\right|+\left|u_{x x x}^{K}\right|+\left|u_{t}^{K}\right| \leq C\left(\ell,\left\|u^{K}\right\|_{L^{\infty}}\right),
$$


with $C$ independent of the truncation domain $K$. By choosing $K$ appropriately, solutions of Eqn. (8.1) are also solutions of Eqn. (1.1). Indeed, if $u^{K}(t, x)$ is a solution of Eqn. (8.1) with $\left|u^{K}(t, x)\right| \leq C_{1}$, then by Eqn. (8.2), $\left|u_{x}^{K}(t, x)\right| \leq C_{2}\left(\ell, C_{1}\right)$. If we choose $K \geq \max \left(C_{1}, C_{2}\right)$, then solutions $u^{K}$ of Eqn. (8.1), with $\left|u^{K}(t, x)\right| \leq C_{1}$, are also solutions of Eqn. (1.1).

For convenience of notation we now omit the superscript $K$. We discretize Eqn. (8.1) as follows: Let $u_{i}(t)=u(t, i / d)$ and

$$
u_{i}^{\prime}=d^{2}\left(u_{i+1}-2 u_{i}+u_{i-1}\right)+g_{K}\left(\frac{i}{d}, u_{i}, d\left(u_{i+1}-u_{i}\right)\right)+\mathcal{E}_{i}^{d}\left(u_{i-1}, u_{i}, u_{i+1}\right),
$$

where $u_{i}^{\prime}$ denotes $\frac{d}{d t} u(t, i / d)$. As before, $\left|\mathcal{E}_{i}^{d}\right| \leq\left|\epsilon_{i}(d)\right| \leq C / d$. The perturbations $\mathcal{E}_{i}^{d}$ are chosen such that the given stationary solutions of Eqn. (1.1) are also discretized solutions of Eqn. (8.3).

Let $\left\{u_{i}^{d}(t)\right\}$ be a sequence of solutions to Eqn. (8.3) with $\left|u_{i}^{d}(t)\right| \leq C_{1}$ for all $i$ and $d$. We will show that one can pass to the limit as $d \rightarrow \infty$ and obtain a complete solution to Eqn. (1.1). The following lemma is proved in a manner analogous to that of Lemma 7.3 of $\$ 7$

\section{Lemma 8.1.}

$$
\int_{J} \sum_{i} \frac{1}{d}\left|\Delta u_{i}\right|^{2} d t \leq C
$$

where $J$ denotes the time interval $[T, T+1]$, and $C$ is independent of $K$.

Proof. If we multiply Eqn. (8.3) by $u_{i}$ and then sum over $i=0, \ldots, d$ and integrate over $t \in[T, T+1]$ we obtain the desired estimate as in Appendix B. This uses the growth of $g$ in $u_{x}$ given by hypothesis (g1).

Fix $K \geq \max \left(C_{1}, C_{2}\right)$, with $C_{1}$ and $C_{2}$ as above, and let $f_{i}=g_{K}+\mathcal{E}_{i}^{d}$. Then

$$
\int_{J} \sum_{i} \frac{1}{d}\left|f_{i}\right|^{2} d t \leq C
$$

Write each solution $u_{i}(t)$ as a sum of terms $u_{i}=u_{i}^{h}+u_{i}^{p}$, where

$$
\begin{aligned}
\frac{d}{d t} u_{i}^{h}-\Delta^{2} u_{i}^{h} & =0, & u_{i}^{h}(T) & =u_{i}(T), \\
\frac{d}{d t} u_{i}^{p}-\Delta^{2} u_{i}^{p} & =f_{i}, & u_{i}^{p}(T) & =0 .
\end{aligned}
$$

Then, for the homogeneous solutions $u_{i}^{h}$, one estimates

$$
\int_{J^{\prime}} \frac{1}{d} \sum_{i}\left|\Delta^{2} u_{i}^{h}\right|^{2} \leq \frac{C}{d} \sum_{i}\left|u_{i}(T)\right|^{2} \leq C, \quad J^{\prime}=[T+\delta, T+1] .
$$

This leads to the following estimate:

$$
\int_{J^{\prime}} \frac{1}{d} \sum_{i}\left|\left(u_{i}^{h}\right)^{\prime}\right|^{2} d t+\int_{J^{\prime}} \frac{1}{d} \sum_{i}\left|\Delta^{2} u_{i}^{h}\right|^{2} d t \leq C .
$$

For the particular solution $u_{i}^{p}$, we have

$$
\frac{1}{d} \sum_{i}\left|f_{i}\right|^{2}=\frac{1}{d} \sum_{i}\left|\left(u_{i}^{p}\right)^{\prime}\right|^{2}-\frac{2}{d} \sum_{i}\left(u_{i}^{p}\right)^{\prime} \Delta^{2} u_{i}+\frac{1}{d} \sum_{i}\left|\Delta^{2} u_{i}^{p}\right|^{2}
$$


For the middle term on the right hand side we have the identity $-\frac{2}{d} \sum_{i}\left(u_{i}^{p}\right)^{\prime} \Delta^{2} u_{i}=$ $\frac{d}{d t} \sum_{i} \frac{1}{d}\left|\Delta u_{i}^{p}\right|^{2}$. Upon integration over $J=[T, T+1]$ we obtain

$$
\int_{J} \frac{d}{d t} \sum_{i} \frac{1}{d}\left|\Delta u_{i}^{p}\right|^{2} d t=\left.\frac{1}{d} \sum_{i}\left|\Delta u_{i}^{p}\right|_{T}^{T+1}\right|^{2}=\frac{1}{d} \sum_{i}\left|\Delta u_{i}^{p}(T+1)\right|^{2} \geq 0 .
$$

Combining these, we obtain

$$
\int_{J} \frac{1}{d} \sum_{i}\left|\left(u_{i}^{p}\right)^{\prime}\right|^{2} d t+\int_{J} \frac{1}{d} \sum_{i}\left|\Delta^{2} u_{i}^{p}\right|^{2} d t \leq \int_{J} \frac{1}{d} \sum_{i}\left|f_{i}\right|^{2} d t \leq C .
$$

Combining the latter with the similar estimate for $u_{i}^{h}$ gives the following estimate for the sum $u_{i}=u_{i}^{p}+u_{i}^{h}$ :

$$
\int_{J^{\prime}} \frac{1}{d} \sum_{i}\left|\left(u_{i}\right)^{\prime}\right|^{2} d t+\int_{J^{\prime}} \frac{1}{d} \sum_{i}\left|\Delta^{2} u_{i}\right|^{2} d t \leq C .
$$

Introduce the spline interpolation

$$
\begin{aligned}
\operatorname{sP}\left(u_{i}\right) & =d \Delta^{2} u_{i+1}(x-i / d)^{3}-\Delta^{2} u_{i+1}(x-i / d)^{2} \\
& +\Delta u_{i}(x-i / d)+u_{i} .
\end{aligned}
$$

Now set $\widetilde{U}^{d}=\operatorname{SP}\left(u_{i}\right)$, and $U^{d}=\operatorname{PL}\left(u_{i}\right)=\Delta u_{i}(x-i / d)+u_{i}$. Then,

$$
\begin{aligned}
\int_{J^{\prime}} \int_{S^{1}}\left|\widetilde{U}^{d}-U^{d}\right|^{2} d x d t & \leq \frac{C}{d^{4}} \rightarrow 0, \quad \text { as } d \rightarrow \infty, \\
\int_{J^{\prime}} \int_{S^{1}}\left|\widetilde{U}_{x}^{d}-U_{x}^{d}\right|^{2} d x d t & \leq \frac{C}{d^{2}} \rightarrow 0, \quad \text { as } d \rightarrow \infty, \\
\int_{S^{1}}\left|\widetilde{U}_{x x}^{d}\right|^{2} d x & \leq C \sum_{i} \frac{1}{d}\left|\Delta^{2} u_{i}\right|^{2}, \\
\int_{S^{1}}\left|\widetilde{U}_{t}^{d}\right|^{2} d x & \leq C \sum_{i} \frac{1}{d}\left|u_{i}^{\prime}\right|^{2} .
\end{aligned}
$$

From the latter two inequalities we derive that

$$
\widetilde{U}^{d} \in H^{1,2}\left(J^{\prime} ; L^{2}\left(S^{1}\right)\right) \cap L^{2}\left(J^{\prime} ; H^{2,2}\left(S^{1}\right)\right) \subset C\left(J^{\prime} ; H^{1,2}\left(S^{1}\right)\right),
$$

which implies that $\sum_{i} \frac{1}{d}\left|\Delta u_{i}(t)\right|^{2} \leq C \quad \forall t \in \mathbb{R}$. Moreover,

$$
\begin{aligned}
& \widetilde{U}^{d}, U^{d} \rightarrow u, \quad \text { in } L^{2}\left(J^{\prime} ; H^{1,2}\left(S^{1}\right)\right), \\
& \widetilde{U}_{t}^{d}, U_{t}^{d} \rightarrow u_{t} \quad \text { in } L^{2}\left(J^{\prime} ; L^{2}\left(S^{1}\right)\right) .
\end{aligned}
$$

From these embeddings one easily deduces that

$$
g_{K}\left(x, U^{d}, U_{x}^{d}\right) \longrightarrow g_{K}\left(x, u, u_{x}\right) \text { in } L^{2}\left(J^{\prime} ; L^{2}\left(S^{1}\right)\right) .
$$

Choose smooth test functions of the form $\phi(t, x)=\sum_{k=1}^{N} \alpha_{k}(t) w_{k}(x)$, where $\left\{w_{k}\right\}$ is an orthonormal basis for $H^{1,2}\left(S^{1}\right)$. Set $\phi_{i}(t)=\phi(t, i / d)$, and $\Phi^{d}=\operatorname{PL}\left(\phi_{i}\right)$. Then

$$
\int_{J^{\prime}} \sum_{i} \frac{1}{d}\left(g_{K}\left(i / d, u_{i}, \Delta u_{i}\right)+\mathcal{E}_{i}^{d}\right) \phi_{i} d t \longrightarrow \int_{J^{\prime}} \int_{S^{1}} g_{K}\left(x, u, u_{x}\right) \phi d x d t .
$$


Because of the PL approximation the following integrals become sums over the anchor points:

$$
\begin{aligned}
& \int_{S^{1}} U_{x}^{d} \Phi_{x} d x=\sum_{i} \frac{1}{d} \Delta u_{i} \Delta \phi_{i}=-\sum_{i} \frac{1}{d} \Delta^{2} u_{i} \phi_{i}, \\
& \int_{S^{1}} U_{t}^{d} \Phi d x=\sum \frac{1}{d} u_{i}^{\prime} \phi_{i}+\frac{1}{3 d} \sum_{i} \frac{1}{d}\left(u_{i+1}^{\prime}-u_{i}^{\prime}\right) \Delta \phi_{i}, \\
& \int_{S^{1}} f \Phi d x=\sum_{i} \frac{1}{d} f_{i} \phi_{i}+\frac{1}{2 d} \sum_{i} \frac{1}{d} f_{i} \Delta \phi_{i} .
\end{aligned}
$$

The final terms of the last two equations admit the following bounds:

$$
\begin{aligned}
\left|\frac{1}{3 d} \sum_{i} \frac{1}{d}\left(u_{i+1}^{\prime}-u_{i}^{\prime}\right) \Delta \phi_{i}\right| & \leq \frac{2}{3 d}\left(\int_{J} \sum_{i} \frac{1}{d}\left|f_{i}\right|^{2} d x\right)^{\frac{1}{2}}\left(\int_{J} \sum_{i} \frac{1}{d}\left|\Delta \phi_{i}\right|^{2} d x\right)^{\frac{1}{2}} \\
& \leq \frac{C}{d} \rightarrow 0, \\
\frac{1}{2 d} \sum_{i} \frac{1}{d} f_{i} \Delta \phi_{i} & \leq \frac{1}{2 d}\left(\int_{J} \sum_{i} \frac{1}{d}\left|u_{i}^{\prime}\right|^{2} d x\right)^{\frac{1}{2}}\left(\int_{J} \sum_{i} \frac{1}{d}\left|\Delta \phi_{i}\right|^{2} d x\right)^{\frac{1}{2}} \\
& \leq \frac{C}{d} \rightarrow 0 .
\end{aligned}
$$

Weak convergence implies that as $d \rightarrow \infty$,

$$
\begin{gathered}
\int_{J} \int_{S^{1}}\left[U^{d} \Phi+U_{x}^{d} \Phi_{x}\right] d x d t \\
\int_{J} \int_{S^{1}} U_{t}^{d} \Phi d x d t \int_{J}\left[u \phi+u_{x} \phi_{x}\right] d x d t, \\
\int_{J} \int_{S^{1}} u_{t} \phi d x d t
\end{gathered}
$$

where $u(t, x)$ is the weak limit of $U^{d}(t, x)$. Hence, $u$ is a weak solution to Eqn. (8.1) for all smooth test function $\phi$ defined above. These functions form a dense subset in $H^{1,2}\left(J^{\prime} \times S^{1}\right)$, and therefore, since $u_{i}$ satisfies Eqn. (8.3),

$$
\int_{S^{1}} u_{t} \phi d x+\int_{S^{1}} u_{x} \phi_{x} d x=\int_{S^{1}} g_{K}\left(x, u, u_{x}\right) \phi d x, \quad \forall \phi \in H^{1,2}\left(S^{1}\right) .
$$

Standard regularity theory arguments then yield strong solutions to Eqn. (8.1). Then using the $L^{\infty}$-bounds on $u$ we also conclude that $u$ is a weak solution to Eqn. (1.1). Again by using standard regularity techniques one can show that the convergence is in $H^{1,2}\left(J^{\prime} \times S^{1}\right)$. This completes the proof of the following theorem:

Theorem 8.2. For any sequence of bounded solutions $\left\{u_{i}^{d}(t)\right\}$ of Eqn. (8.3) with $\left|u_{i}^{d}(t)\right| \leq C$, for all $t$ and $i, \operatorname{PL}\left(u_{i}^{d}\right)$ converges, in $H^{1,2}\left(J \times S^{1}\right)$, to a (strong) solution $u$ of Eqn. (8.1). Moreover, if $K$ is chosen large enough, then $u$ is a (strong) solution of Eqn. (1.1).

8.2. Proof of Theorem 1.10. Let $\{\mathbf{u}$ REL $\mathbf{v}\}$ be a braid class that does not permit stationary solutions for Eqn. (1.1). For $d$ large enough the same holds for Eqn. (8.3); otherwise, the results in 97 would yield stationary solutions of Eqn. (1.1), a contradiction. If $\{\mathbf{u}$ REL $\mathbf{v}\}$ is bounded and proper with $\mathbf{H}(\mathbf{u}$ REL $\mathbf{v}) \neq 0$, then for each $d$ large enough there exists a periodic solution $\mathbf{u}^{d}$ with strands $u_{i}^{\alpha, d}(t)$ via [22, Thm. 2]. By Theorem 8.2 this sequence yields a solution $u(t, x)$ of Eqn. (1.1). 
It remains to be shown that $u(t, x)$ is periodic in $t$. This follows from the celebrated Poincaré-Bendixson Theorem for scalar parabolic equations due to Fiedler and Mallet-Paret [15, which states that a bounded solution $u(t, x)$ has forward limit set either a stationary point or a time-periodic orbit. By assumption, $\{\mathbf{u}$ REL v $\}$ contains no stationary points, which leaves the second option: a periodic solution. This also proves then that $\{\mathbf{u}$ REL $\mathbf{v}\}$ contains a periodic solution of the desired braid class.

We remark that the proof above is for braid classes $\{\mathbf{u}$ REL $\mathbf{v}\}$ for which $\mathbf{u}$ has a single component. For $\mathbf{u}$ with multiple components, a nonvanishing index implies that each component of $\mathbf{u}$ is either stationary or periodic; however, unless the periods are rationally related, the entire braid class will be merely quasi-periodic as opposed to periodic.

\section{Conclusions}

9.1. Comments. On forcing. The goal of this paper is distinct from the existing literature on lap-number methods for Eqn. (1.1), e.g. [15, 17, 18, 19, 16], in that we focus on forcing on individual stationary or periodic orbits. Our methods are not used with the goal of characterizing the global attractor. We are elucidating the hierarchy of orbits within the attractor via index-theoretic methods.

On topology. The focus of this work is on the inherent topological structure of the invariant sets. This structure is revealed by means of the homotopy braid index. The principal mathematical result of the paper - that the index is a topological invariant of relative braids - has the important implication that there is a dynamical forcing partial order on braids which is completely independent of the analytical details of the equation. Unlike most papers which analyze Eqn. (1.1), we assume neither transversality nor genericity conditions. Spatial inhomogeneity is perfectly acceptable, even in applications to the existence of time-periodic orbits. This is a stark departure from analytic methods [5, 16], which are efficacious in the setting of rigid rotating waves. The topological approach finds non-rigidly-rotating waves.

On computability. The technically challenging portion of this paper is the convergence of the index to the infinite-dimensional setting of parabolic PDEs. With this comes the corollary that the homotopy braid index can be computed from a finite-dimensional model. Since there are explicit bounds on this dimension (in terms of the length of the braid implicated), it is possible to compute the homological braid index using existing software packages. This is an implication of our theory not explored in this work, but nevertheless significant.

9.2. Extensions. Boundary conditions. We have employed periodic boundary conditions for convenience and as a means to allow for time-periodic orbits. Nothing prevents us from using other boundary conditions, although the resulting dynamics is often gradient-like. Neumann, Dirichlet, or (nonlinear) combinations of the two are imposed by choosing closed subsets $B_{0} \subset\left\{\left(0, u, u_{x}\right)\right\}$ and $B_{1} \subset\left\{\left(1, u, u_{x}\right)\right\}$ and requiring the braid endpoints to remain in these subspaces. As the topology of the configuration spaces of braids may change, so may the resulting invariants. Since the comparison principle still holds, our topological methods remain valid, though the invariants themselves may change.

Improper braids. A braid class is improper if components of the braid can be collapsed. Our results on $t$-periodic solutions in $\$ 6$ dealt with improper braids in 
an ad hoc manner by "blowing up" the collapsible strands via adding additional strands to the skeleton.

A different approach would be to blow up the vector field in the traditional manner via homogeneous coordinates, working in the setting of finite-dimensional parabolic recurrence relations. Stabilization then allows one to define the invariant in the continuous limit. This type of blow-up procedure is very general and should be applicable to a wide variety of systems.

p-Laplacians and degenerate parabolic equations. In Remark 1.3 we explained that the results for obtaining stationary solutions of the fully nonlinear equation

$$
u_{t}=f\left(x, u, u_{x}, u_{x x}\right)
$$

follow directly from the semilinear Eqn. (1.1). This is under the assumption of uniform parabolicity, i.e. $0<\lambda \leq \partial_{w} f(x, u, v, w) \leq \lambda^{-1}$. The theory in this paper should also apply to stationary solutions of degenerate parabolic equations of various kinds. One example of a degenerate equation is the 1-dimensional $p$-Laplacian equation $u_{t}=\left(\left|u_{x}\right|^{p-1} u_{x}\right)_{x}+g\left(x, u, u_{x}\right)$. In order to deal with this equation one can consider $u_{t}=\epsilon u_{x x}+\left(\left|u_{x}\right|^{p-1} u_{x}\right)_{x}+g\left(x, u, u_{x}\right)$. After discretization one again obtains a proper parabolic system. With the appropriate a priori estimates involving $p$ one then lets $\epsilon \rightarrow 0$ as the discretization size goes to zero. One avoids intersection principles for the $p$-Laplacian equation since the discrete equation obtained this way is parabolic.

The following estimates, though necessary, are rather antithetical to our philosophy: the entire forcing theory for Eqn. (1.1) is topological in nature.

\section{Appendix A. Estimates: Stationary}

A stationary solution of Eqn. (1.1) is some $u \in C^{2}(\mathbb{R} / \ell \mathbb{Z})$ satisfying $u_{x x}+$ $g\left(x, u, u_{x}\right)=0$. Hypothesis (g1) permits the following regularity statement.

Lemma A.1. Let $u \in C^{2}(\mathbb{R} / \ell \mathbb{Z})$ be a stationary solution of Eqn. (1.1) with $g$ satisfying (g1). There exists a constant $C=C\left(\ell,\|u\|_{L^{\infty}}\right)$ depending only on the sup norm of $u$, such that

$$
\left|u_{x}\right|+\left|u_{x x}\right|+\left|u_{x x x}\right| \leq C .
$$

Proof. Multiply Eqn. (1.1) by $u$. Integrating over $S^{1}:=\mathbb{R} / \ell \mathbb{Z}$, using hypothesis (g1) yields

$$
\begin{aligned}
\int_{S} u_{x}^{2} d x & =\int_{S}|u| \cdot\left|g\left(x, u, u_{x}\right)\right| d x \\
& \leq\|u\|_{L^{\infty}} \int_{S}\left|g\left(x, u, u_{x}\right)\right| d x \\
& \leq C\left(1+\int_{S}\left|u_{x}\right|^{\gamma} d x\right)
\end{aligned}
$$


Since $\gamma<2$, it follows that $\int_{S}\left|u_{x}\right|^{2} d x \leq C$. Again by using hypothesis (g1) we obtain

$$
\begin{aligned}
\int_{S}\left|u_{x x}\right|^{\frac{2}{\gamma}} & =\int_{S}\left|g\left(x, u, u_{x}\right)\right|^{\frac{2}{\gamma}} d x \\
& \leq\left.\left.\int_{S}|C+C| u_{x}\right|^{\mid}\right|^{\frac{2}{\gamma}} d x \\
& \leq C\left(1+\int_{S}\left|u_{x}\right|^{2} d x\right) \leq C .
\end{aligned}
$$

The latter implies that $\|u\|_{W^{2,} \frac{2}{\gamma}} \leq C$. From the Sobolev embeddings for $W^{2, \frac{2}{\gamma}}(S)$ we derive

$$
\|u\|_{C^{1, \alpha}(S)} \leq C\|u\|_{W^{2, \frac{2}{\gamma}}} \leq C,
$$

with $0<\alpha<1-\frac{\gamma}{2}<1$. In particular $\left\|u_{x}\right\|_{L^{\infty}} \leq C$. Again by using the pointwise bound $\left|u_{x x}\right| \leq\left|g\left(x, u, u_{x}\right)\right|$ we obtain

$$
\begin{aligned}
\sup _{x}\left|u_{x x}\right| & \leq\left\|g\left(x, u, u_{x}\right)\right\|_{L^{\infty}} \\
& \leq C+C\left\|u_{x}\right\|_{L^{\infty}}^{\gamma} \leq C,
\end{aligned}
$$

which implies that $\left\|u_{x x}\right\| \leq C$. By differentiating the equation and using the fact that $g \in C^{1}$ to estimate $u_{x x x}$, we obtain

$$
\partial_{x} g+\partial_{u} g \cdot u_{x}+\partial_{u_{x}} g \cdot u_{x x}+u_{x x x}=0
$$

For $u_{x x x}$ this yields

$$
\left|u_{x x x}\right| \leq\left|\partial_{x} g\right|+\left|\partial_{u_{x}} g\right|\left|u_{x}\right|+\left|u_{x x}\right| \leq C,
$$

since all derivatives of $f$ can be bounded in terms of $\|u\|_{L^{\infty}}$. This completes the proof.

\section{Appendix B. Estimates: Nonstationary}

We repeat the regularity arguments for nonstationary solutions to Eqn. (1.1). As the estimates are similar in spirit as those of Appendix A, we omit the more unseemly steps.

Lemma B.1. Let $u \in C^{1}\left(\mathbb{R} ; C^{2}(\mathbb{R} / \ell \mathbb{Z})\right)$ be a complete bounded solution with $g$ satisfying (g1). There exists a constant $C=C\left(\ell,\|u\|_{L^{\infty}}\right)$ depending only on the sup norm of $u(t, x)$, such that

$$
\left|u_{x}\right|+\left|u_{x x}\right|+\left|u_{x x x}\right|+\left|u_{t}\right| \leq C .
$$

Proof. As before, let $S^{1}:=\mathbb{R} / \ell \mathbb{Z}$. Denote by $J$ the time interval $J:=[T, T+1]$. Multiplying Eqn. (1.1) by $u$ and integrating by parts yields

$$
\int_{J} \int_{S^{1}} u_{t} u d x d t=-\int_{J} \int_{S^{1}} u_{x}^{2} d x d t+\int_{J} \int_{S^{1}} g\left(x, u, u_{x}\right) u d x d t .
$$

Using hypothesis (g1) we derive

$$
\int_{J} \int_{S^{1}} u_{x}^{2} d x d t \leq-\left.\frac{1}{2} \int_{S^{1}} u^{2} d x\right|_{T} ^{T+1}+C+C \int_{J} \int_{S^{1}}\left|u_{x}\right|^{\gamma} d x d t .
$$

Hence, since $\gamma<2, \int_{J} \int_{S^{1}}\left|u_{x}\right|^{2} d x d t \leq C$. 
We proceed with the more technical estimates. Given the solution $u(t, x)$,

$$
u_{t}-u_{x x}=g\left(x, u(t, x), u_{x}(t, x)\right) \in L^{\frac{2}{\gamma}}\left(J ; L^{\frac{2}{\gamma}}\left(S^{1}\right)\right),
$$

since $|g|^{2 / \gamma} \leq C+C\left|u_{x}\right|^{2}$. As such, $L^{p}$ regularity theory implies (see, e.g., [8])

$$
\begin{aligned}
\left\|u_{t}\right\|_{L^{\frac{2}{\gamma}}\left(J^{\prime} ; L^{\frac{2}{\gamma}}\left(S^{1}\right)\right)} & \leq C(\delta)\|g\|_{L^{\frac{2}{\gamma}\left(J ; L^{\frac{2}{\gamma}}\right)}}, \\
\left\|u_{x x}\right\|_{L^{\frac{2}{\gamma}}\left(J^{\prime} ; L^{\frac{2}{\gamma}}\left(S^{1}\right)\right)} & \leq C(\delta)\|g\|_{L^{\frac{2}{\gamma}\left(J ; L^{\frac{2}{\gamma}}\right)},},
\end{aligned}
$$

where $J^{\prime}:=[T+\delta, T] \subset J$ for some $0<\delta \ll 1$. In particular,

$$
u \in L^{\frac{2}{\gamma}}\left(J^{\prime} ; H^{2, \frac{2}{\gamma}}\left(S^{1}\right)\right) \cap L^{\infty}\left(\mathbb{R} ; L^{\infty}\left(S^{1}\right)\right) .
$$

Bootstrapping proceeds in a standard fashion using a parabolic version of the Gagliardo-Nirenberg interpolation inequalities. Given any function $u \in$ $L^{p}\left(J^{\prime}, H^{2, p}\left(S^{1}\right)\right) \cap L^{\infty}\left(J^{\prime}, L^{\infty}\left(S^{1}\right)\right)$, then

$$
\|u\|_{L^{2 p}\left(J^{\prime} ; H^{1,2 p}\left(S^{1}\right)\right)} \leq C\|u\|_{L^{p}\left(J^{\prime}, H^{2, p}\right)}^{\frac{1}{2}} \cdot\|u\|_{L^{\infty}\left(J^{\prime}, L^{\infty}\right)}^{\frac{1}{2}} .
$$

Therefore, we have $u \in L^{\frac{4}{\gamma}}\left(J^{\prime}, H^{1, \frac{4}{\gamma}}\left(S^{1}\right)\right)$ and, hence, $g \in L^{\frac{4}{\gamma^{2}}}\left(J^{\prime} ; L^{\frac{4}{\gamma^{2}}}\left(S^{1}\right)\right)$.

We repeat the procedure $k$ times, each time restricting the time domain $[T+$ $k \delta, T+1]$. Choose $k>0$ sufficiently large so that $(2 / \gamma)^{k}>2$ and choose $\delta$ sufficiently small so that $[T+k \delta, T+1]$ contains $J^{\prime \prime}:=\left[T+\frac{1}{2}, T+1\right]$. Then we have

$$
u \in H^{1,2}\left(J^{\prime \prime} ; L^{2}\left(S^{1}\right)\right) \cap L^{2}\left(J^{\prime \prime} ; H^{2,2}\left(S^{1}\right)\right) .
$$

By Sobolev embedding, we get $u \in C\left(J^{\prime \prime} ; H^{1,2}\left(S^{1}\right)\right)$. Repeating the entire procedure yields $u \in C^{\alpha}\left(J^{\prime \prime} ; C^{1, \alpha}\left(S^{1}\right)\right)$. This bound is now independent of $T$, and one translates to obtain $u \in C^{\alpha}\left(\mathbb{R} ; C^{1, \alpha}\left(S^{1}\right)\right)$. The additional smoothness now follows directly from the fact that $u$ solves Eqn. (1.1).

The $C^{3}$-estimate is obtained as in the stationary case by differentiating the equation and using the $C^{1,2}$-estimates obtained above.

\section{Appendix C. Discrete enclosure}

Using a discrete version of enclosure between sub/super solutions and a nontrivial braid diagram, we obtain the following existence result.

Lemma C.1. Let $g$ satisfy hypothesis (g1) and let $\mathbf{v}$ be a nontrivially braided stationary braid for Eqn. (1.1). Assume that there exists a $u^{*}$ such that $v^{\alpha}(x)<u^{*}$ for all $\alpha$ and $g\left(x, u^{*}, 0\right)<0$. Then, there exists a 1-periodic solution $u$ with

$$
\max _{\alpha} v^{\alpha}(x)<u(x)<u^{*},
$$

for all $x \in S^{1}$.

It is clear that the same result also holds for the case of a $u^{*}$ such that $u^{*}<v^{\alpha}(x)$ for all $\alpha$ and $g\left(x, u^{*}, 0\right)>0$. In that case one finds a solution $u$ satisfying

$$
u^{*}<u(x)<\min _{\alpha} v^{\alpha}(x)
$$

for all $x \in S^{1}$. 
Proof. As in $\$ 7$ we discretize Eqn. (1.1) in $x$. For $u^{*}$ this implies that $g\left(i / d, u^{*}, 0\right)<$ 0 . As for the braid $\mathbf{v}$ we use Lemma 7.2 to find $\mathcal{E}_{i}^{d}$ and the recurrence relation $\mathcal{R}_{i}^{d}\left(u_{i-1}, u_{i}, u_{i+1}\right):=\Delta^{2} u_{i}+g\left(i / d, u_{i}, \Delta u_{i}\right)+\mathcal{E}_{i}^{d}\left(u_{i-1}, u_{i}, u_{i+1}\right)$. By construction the discretized skeleton $\operatorname{DISC}_{d} \mathbf{v}$ is stationary for $\mathcal{R}$.

Define the region

$$
D=\left\{\left\{u_{i}\right\}_{i=0}^{d} \mid \max _{\alpha} v_{i}^{\alpha} \leq u_{i} \leq u^{*}, u_{0}=u_{d}\right\} .
$$

If the discretization is chosen fine enough, then the discretized braid is nontrivial. As a consequence $u_{i}$ cannot collapse onto $\operatorname{DISC}_{d} \mathbf{v}$ and if $u_{i}=v_{i}^{\alpha}$ for some $i$ and some $\alpha$, then $\mathcal{R}_{i}^{d}\left(u_{i-1}, u_{i}, u_{i+1}\right)>0$. By the definition of $u^{*}$ it follows that if $u_{i}=u^{*}$ for some $i$, then $\mathcal{R}_{i}^{d}\left(u_{i-1}, u_{i}, u_{i+1}\right) \leq g\left(i / d, u_{i}, 0\right)<0$ (parabolicity). The region $D$ is therefore an attracting isolating (compact) set for Eqn. (4.2). Thus for each large enough $d$ we find a discrete solution $\left\{u_{i}^{d}\right\}_{i=0}^{d}$. Since $\left\{u_{i}^{d}\right\}_{i=0}^{d}$ is a priori bounded we derive from the limiting procedure in $\$ 7$ that this yields a stationary solution $u(x)$ for Eqn. (1.1), satisfying the desired inequality.

\section{Appendix D. Proof of Theorem 1.5}

In order to prove Theorem 1.5 we employ a different discretization scheme which allows an easy proof of multiplicity. The drawback of this approach is that it cannot be used in the nonstationary case. The approach used in $\$ 7$ and $\$ 8$ works for both the stationary and nonstationary cases.

Consider the boundary value problem

$$
\begin{array}{r}
u_{x x}+g(x, u)=0, \quad x \in\left(x_{i}, x_{i+1}\right), \\
u\left(x_{i}\right)=u_{i}, \quad u\left(x_{i+1}\right)=u_{i+1},
\end{array}
$$

where $x_{i}=i / d$, and $i \in\{0, \ldots, d\}$. Solutions of Eqn. (D.1) are stationary points of the action integral $\int_{x_{i}}^{x_{i+1}} L\left(x, u, u_{x}\right) d x$ over functions $u \in H^{1}\left(x_{i}, x_{i+1}\right)$ with boundary conditions $u\left(x_{i}\right)=u_{i}$ and $u\left(x_{i+1}\right)=u_{i+1}$. For Eqn. (1.3) the Lagrangian is given by $L\left(x, u, u_{x}\right)=\frac{1}{2}\left|u_{x}\right|^{2}+G(x, u)$, with $\partial_{u} G(x, u)=g(x, u)$. Since the relative braid class $\{\mathbf{u}$ REL $\mathbf{v}\}$ is bounded, we have two strands $v^{-}, v^{+} \in \mathbf{v}$ such that $v^{-}<u^{\alpha}<v^{+}$for all $u^{\alpha} \in \mathbf{u}$. Define the strips

$$
D_{x}=\left\{u \mid v^{-}(x)<u<v^{+}(x)\right\} .
$$

Minimization of the action integral yields the following existence result.

Lemma D.1. There exists a $d_{0}$ such that for all $d \geq d_{0}$, Eqn. (D.1) has a solution $u\left(x ; u_{i}, u_{i+1}\right)$ for all $\left(u_{i}, u_{i+1}\right) \in D_{x_{i}} \times D_{x_{i+1}}$, and all $i$. Moreover, $u\left(x, u_{i}, u_{i+1}\right) \in$ $D_{x}$ for all $x \in\left[x_{i}, x_{i+1}\right]$.

Proof. Minimiize the action $\int_{x_{i}}^{x_{i+1}} L\left(x, u, u_{x}\right) d x$ over functions $u \in H^{1}\left(x_{i}, x_{i+1}\right)$ with boundary conditions $u\left(x_{i}\right)=u_{i}$ and $u\left(x_{i+1}\right)=u_{i+1}$. For $d_{0}$ sufficiently large the functions $v^{ \pm}$are minimizers for the action. Therefore, via a standard cutting and pasting argument, we can minimize the action over functions $u$ that satisfy $v^{-} \leq u \leq v^{+}$. Indeed, if $u$ intersects $v^{ \pm}$, cutting out the graph above/below $v^{ \pm}$ and pasting in the particular part of $v^{ \pm}$, decreases the action.

It is clear that for any $d$ the action is bounded from below on the functions described above. Now a standard minimization argument completes the proof. 
Uniqueness of these solutions can be concluded by studying the following initial value problem:

$$
\begin{array}{r}
v_{x x}+g(x, v)=0, \quad x \in(0,1), \\
v\left(x_{i}\right)=u_{i}, \quad v_{x}\left(x_{i}\right)=p_{i} .
\end{array}
$$

The dependence of $v\left(x ; u_{i}, p_{i}\right)$ on $p_{i}$ yields the property that $\partial_{p_{i}} v\left(x_{i+1} ; u_{i}, p_{i}\right)>0$ for all $d \geq d_{0}$. Indeed, by linearizing Eqn. (D.2), we obtain the equation

$$
\phi_{x x}+a(x) \phi=0
$$

with boundary conditions $\phi\left(x_{i}\right)=0$, and $\phi_{x}\left(x_{i}\right)=1$, and $a(x)=\partial_{v} g(x, v)$. By construction, $\partial_{p_{i}} v\left(x_{i+1} ; u_{i}, p_{i}\right)=\phi\left(x_{i+1}\right)$. Rescale $x$ as $x=x_{i}+y / d$. Then $\phi_{y y}+$ $\frac{\widehat{a}(y)}{d^{2}} \phi=0, y \in(0,1)$, and where $\widehat{a}(y)=a\left(x_{i}+y / d\right)$.

Now pick any solution $u\left(x ; u_{i}, u_{i+1}\right)$ of Eqn. (D.1) and consider Eqn. (D.2) with $p_{i}=u_{x}\left(x_{i}\right)$. Then, since $u(x) \in D_{x}$ for $x \in\left[x_{i}, x_{i+1}\right]$, it holds that $a(y)$ is uniformly bounded by some constant $C$ independent of $d$. Therefore, there exists a $d_{1} \geq d_{0}$ such that $\left.\phi\right|_{y=1}>0$, which proves our claim. This monotonicity now prevents Eqn. (D.1) from having a second solution, thus proving uniqueness.

If we concatenate solutions $u\left(x ; u_{i}, u_{i+1}\right)$ on $(0,1)$ with the boundary condition $u_{0}=u_{d}$, then the concatenation is a solution of Eqn. (D.1) except at the 'glue points' $x_{i}$. The concatenation can be referred to as a broken geodesic. The condition of being an actual smooth solution of Eqn. (D.1) then reads

$$
\mathcal{R}_{i}\left(u_{i-1}, u_{i}, u_{i+1}\right)=u_{x}\left(x_{i} ; u_{i}, u_{i+1}\right)-u_{x}\left(x_{i-1} ; u_{i-1}, u_{i}\right)=0 .
$$

Uniqueness of the solutions on $\left(x_{i}, x_{i+1}\right)$ yields the relation

$$
v\left(x_{i+1} ; u_{i}, u_{x}\left(x_{i} ; u_{i}, u_{i+1}\right)\right)=u_{i+1} .
$$

Upon differentiating this relation we obtain

$$
1=\partial_{p_{i}} v\left(x_{i+1} ; u_{i}, u_{x}\left(x_{i} ; u_{i}, u_{i+1}\right)\right) \cdot \partial_{u_{i+1}} u_{x}\left(x_{i} ; u_{i}, u_{i+1}\right),
$$

which implies that $\partial_{u_{i+1}} u_{x}\left(x_{i} ; u_{i}, u_{i+1}\right)>0$. By the same token one proves that $\partial_{u_{i-1}} u_{x}\left(x_{i-1} ; u_{i-1}, u_{i}\right)<0$. These inequalities prove that $\mathcal{R}_{i}$ as defined above is a parabolic recurrence relation for all $d \geq d_{1}$.

The next step is to choose $d$ small enough so that $\{\mathbf{u}$ REL $\mathbf{v}\}$ can be properly represented in the discrete setting. Since the laps on the intervals $\left(x_{i}, x_{i+1}\right)$ are solutions of $u_{x x}+g(x, u)=0$, an ordering of solutions exists.

(i) If $\left(\widetilde{u}_{i}, \widetilde{u}_{i+1}\right)>\left(u_{i}, u_{i+1}\right)$ 5 then $u\left(x ; \widetilde{u}_{i}, \widetilde{u}_{i+1}\right)>u\left(x ; u_{i}, u_{i+1}\right)$ for all $x \in$ $\left(x_{i}, x_{i+1}\right)$.

(ii) If $\left( \pm \widetilde{u}_{i}, \mp \widetilde{u}_{i+1}\right)>\left( \pm u_{i}, \mp u_{i+1}\right)$, then the intersection number for $x \in\left[x_{i}\right.$, $\left.x_{i+1}\right]$ satisfies $\iota\left(u\left(x ; \widetilde{u}_{i}, \widetilde{u}_{i+1}\right), u\left(x ; u_{i}, u_{i+1}\right)\right)=1$.

These two properties are easily derived from the property that two solutions cannot be tangent unless they are identical and the continuous dependence of $\left(u_{i}, u_{i+1}\right)$. The above properties (i) and (ii) imply that for $d \geq d_{1}$ it holds that $\left\{\operatorname{PL}\left(\operatorname{DISC}_{d}(\mathbf{v})\right)\right\}$ $=\{\mathbf{v}\}$. Let $\mathbf{u}$ REL $\mathbf{v}$ be a representative in $\{\mathbf{u}$ REL $\mathbf{v}\}$, and choose $d_{2} \geq d_{1}$ such that for $d \geq d_{2}$. It holds that $\operatorname{PL}\left(\operatorname{DISC}_{d}(\mathbf{u})\right) \operatorname{REL} \operatorname{PL}\left(\operatorname{DISC}_{d}(\mathbf{v})\right) \in\{\mathbf{u} \operatorname{REL} \mathbf{v}\}$. Using the above properties (i) and (ii) again we conclude that solutions of $\mathcal{R}_{i}=0$ in $\left[\operatorname{DISC}_{d}(\mathbf{u})\right.$ REL DISC $\left.d(\mathbf{v})\right]$ indeed yield solutions $u(x)$ of Eqn. (1.1) in the desired braid class $\{\mathbf{u}$ REL $\mathbf{v}\}$. This provides yet another proof of Theorem 1.1 in this special case and does not require a limiting argument in $d$. The multiplicity results in

\footnotetext{
${ }^{5}$ With the ordering $(a, b)>(c, d)$ we indicate that $a \geq c, b \geq d$, and $(a, b) \neq(c, d)$.
} 
22 now imply that $\left|P_{t}(\mathbf{H})\right|$ is a lower bound on the number of discretized braids in $\left[\operatorname{DISC}_{d}(\mathbf{u}) \operatorname{REL} \operatorname{DISC}_{d}(\mathbf{v})\right]$, and therefore also a lower bound for a number of distinct stationary braids in $\{\mathbf{u}$ REL $\mathbf{v}\}$ for Eqn. (1.1).

\section{REFERENCES}

[1] N. Alikakos, P. Bates and G. Fusco, Solutions to the nonautonomous bistable equation with specified Morse index. Part I: Existence. Trans. Amer. Math. Soc. 340(2), 1993, 641-654. MR 1167183 (94b:34032)

[2] S. Angenent, The zero set of a solution of a parabolic equation, J. Reine Angew. Math. 390, 1988, 79-96. MR953678 (89j:35015)

[3] S. Angenent, Curve shortening and the topology of closed geodesics on surfaces, Ann. of Math. (2) 162(3), 2005, 1185-1239. MR2179729 (2006j:53094)

[4] S. Angenent, The periodic orbits of an area preserving twist map, Comm. Math. Phys. 115, 1988, 353-374. MR931667 (89f:58118)

[5] S. Angenent and B. Fiedler, The dynamics of rotating waves in scalar reaction diffusion equations, Trans. Amer. Math. Soc. 307(2), 1988, 545-568. MR940217 (89h:35158)

[6] S. Angenent, J. Mallet-Paret, and L. Peletier, Stable transition layers in a semilinear boundary value problem, J. Diff. Eqs. 67(2), 1987, 212-242. MR879694 (88d:34018)

[7] J. Birman, Braids, links and the mapping class group, Ann. Math. Stud. 82, Princeton Univ. Press, 1975. MR 0375281 (51:11477)

[8] H. Brezis, Analyse Fonctionnelle, Masson, Paris, 1983. MR697382 (85a:46001)

[9] P. Brunovský and B. Fiedler, Connecting orbits in scalar reaction diffusion equations, in Dynamics Reported, Vol. 1, Wiley, Chichester, 1988, 57-89. MR945964 (89j:58071)

[10] P. Brunovský and B. Fiedler, Connecting orbits in scalar reaction diffusion equations II: The complete solution, J. Diff. Eqs., 81(1), 1989, 106-135. MR1012202 (91d:35111)

[11] P. Brunovský and B. Fiedler, Simplicity of zeros in scalar parabolic equations, J. Diff. Eqs., 62(2), 1986, 237-241. MR833419 (88c:35079)

[12] K.C. Chang, Infinite-dimensional Morse Theory and Multiple Solution Problems, Birkhäuser, Boston, 1991. MR 1196690 (94e:58023)

[13] C. Conley, Isolated Invariant Sets and the Morse Index, CBMS Reg. Conf. Ser. Math. 38, Amer. Math. Soc., Providence, RI, 1978. MR.511133 (80c:58009)

[14] N. Dancer, Degenerate critical points, homotopy indices and Morse inequalities, J. Reine Angew. Math. 350, 1984, 1-22. MR743531 (85i:58033)

[15] B. Fiedler and J. Mallet-Paret, A Poincaré-Bendixson theorem for scalar reaction diffusion equations. Arch. Rational Mech. Anal. 107, 1989, no. 4, 325-345. MR 1004714 (90j:35116)

[16] B. Fiedler, C. Rocha, and M. Wolfrum, Heteroclinic orbits between rotating waves of semilinear parabolic equations on the circle, J. Diff. Eqs., 201(1), 2004, 99-138. MR2057540 (2005f:37172)

[17] B. Fiedler and C. Rocha, Orbit equivalence of global attractors of semilinear parabolic differential equations, Trans. Amer. Math. Soc., 352(1), 2000, 257-284. MR1475682|(2000c:35129)

[18] B. Fiedler and C. Rocha, Realization of meander permutations by boundary value problems, J. Diff. Eqs., 156(2), 1999, 282-308. MR1705403 (2001b:37112)

[19] B. Fiedler and C. Rocha, Heteroclinic orbits of semilinear parabolic equations, J. Diff. Eqs., 125(1), 1996, 239-281. MR:1376067 (96k:58200)

[20] G. Fusco and W. Oliva, Jacobi matrices and transversality, Proc. Roy. Soc. Edinburgh Sect. A Math. 109, 1988, 231-243. MR963029 (89k:58229)

[21] R. Ghrist, Braids and differential equations, Proc. International Congress of Mathematicians, vol. III, 2006, 1-26. MR2275668

[22] R. Ghrist, J. B. Van den Berg and R. C. Vandervorst, Morse theory on spaces of braids and Lagrangian dynamics, Invent. Math. 152, 2003, 369-432. MR1974892 (2004e:37025)

[23] D. Gromoll and W. Meyer, On differentiable functions with isolated critical points. Topology 8, 1969, 361-369. MR0246329 (39:7633)

[24] J. Hale, Dynamics of a scalar parabolic equation. Canad. Appl. Math. Quart. 5(3), 1997, 209-305. MR1637218 (2000b:35112)

[25] D. Henry, Geometric Theory of Semilinear Parabolic Equations, Lecture Notes in Mathematics, Vol. 840, Springer, Berlin, 1981. MR610244(83j:35084) 
[26] J. Mallet-Paret and H. Smith, The Poincaré-Bendixson theorem for monotone cyclic feedback systems, J. Dyn. Diff. Equations 2, 1990, 367-421. MR1073471 (91k:58098)

[27] H. Matano, Nonincrease of the lap-number of a solution for a one-dimensional semi-linear parabolic equation, J. Fac. Sci. Tokyo 1A 29 1982, 645-673. MR672070 (84m:35060)

[28] K. Nakashima, Stable transition layers in a balanced bistable equation. Diff. Integral Equations 13, 2000, no. 7-9, 1025-1038. MR1775244 (2001h:34077)

[29] K. Nakashima, Multi-layered stationary solutions for a spatially inhomogeneous Allen-Cahn equation. J. Differential Equations 191, 2003, no. 1, 234-276. MR1973289 (2004f:35097)

[30] E. Séré, Looking for the Bernoulli shift, Ann. Inst. Henri Poincaré 10 (5), 1993, 561-590. MR 1249107 (95b:58031)

[31] J. Smillie, Competitive and cooperative tridiagonal systems of differential equations, SIAM J. Math. Anal. 15, 1984, 531-534. MR740693 (85g:58054)

[32] C. Sturm, Mémoire sur une classe d'équations à différences partielles, J. Math. Pure Appl., 1, 1836, 373-444.

[33] J. B. Van den Berg, R. C. Vandervorst and W. Wójcik, Chaos in orientation preserving twist maps of the plane, Topology and its Applications, 154(13), 2007, 2580-2606. MR2332874 (2008f:37093)

[34] W. Wilson and J. York, Lyapunov functions and isolating blocks, J. Diff. Equations, 13, 1973, 106-123. MR0385251 (52:6115)

[35] T. Zelenyak, Stabilization of solutions of boundary value problems for a second order parabolic equation with one space variable, Differential Equations 4, 1968, 17-22. MR0223758 (36:6806)

Departments of Mathematics and Electrical \& Systems Engineering, University of Pennsylvania, Philadelphia, Pennsylvania 19104

E-mail address: ghrist@math.upenn.edu

Department of Mathematics, Vrije Universiteit Amsterdam, De Boelelaan 1081, 1081 HV, Amsterdam, The Netherlands

E-mail address: vdvorst@ew.vu.nl 